\title{
Frequency-domain least-squares generalized internal multiple imaging with the
}

\section{energy norm}

\author{
Guanchao Wang $^{1 *}$, Qiang Guo ${ }^{2}$, Tariq Alkhalifah ${ }^{2}$, Shangxu Wang ${ }^{1}$ \\ ${ }^{1}$ China University of Petroleum, State Key Laboratory of Petroleum Resources and \\ Prospecting, CNPC Key Laboratory of Geophysical Exploration, Changping 102249, Beijing, \\ China.
}

${ }^{2}$ King Abdullah University of Science and Technology, KAUST.

wgcupc@163.com; qiang.guo@kaust.edu.sa; tariq.alkhalifah@kaust.edu.sa;

wangsx@cup.edu.cn;

*Corresponding author: Guanchao Wang

${ }^{*}$ Corresponding author Email: wgcupc@163.com

Shortened title: Frequency-Domain LS-GIMI with the Energy norm

Keywords: Internal Multiples, Imaging, Frequency-domain, Energy norm 


\begin{abstract}
Recorded seismic data contain various types of scattered energy, including those corresponding to multiples. Traditional imaging techniques are focused on the singlescattering events, and thus, may fail to image crucial structures, such as salt flanks and faults that sometimes are only illuminated by the multiple scattered energy. The recently introduced generalized internal multiple imaging (GIMI) offers an opportunity to image multiples by projecting the recorded data back into the subsurface, followed by an interferometric cross-correlation of the subsurface wavefield with the recorded data. During this process, the interferometric step converts the first-order scattering to a tomographic component and the double-scattering forms the primary reflectivity. Dealing with a large volume of data consisting of full wavefields over the image space, renders the interferometric step computationally expensive in time-domain. To make the implementation of GIMI tractable, we formulate its frequency-domain version. Moreover, we use the energy norm imaging condition to separate the reflectivity part from the tomographic component. We demonstrate these features with numerical experiments.
\end{abstract}

\title{
INTRODUCTION
}

Seismic imaging provides crucial information about the Earth's subsurface structure and an important role in the traditional seismic processing. Most of the popular seismic migration methods, including reverse time migration (RTM), focus only on imaging 
the primary reflections (Claerbout, 1971). The implementation of RTM can be generally described in two steps. First, we forward extrapolate the source wavefield and backpropagate the recorded data. Then, the imaging condition is applied using the two wavefields to extract locations of wavefield continuation, referred to as the reflectivity. Through such a mechanism, RTM can only accurately image single-scattering reflections between the two wavefields. Multiples are usually misplaced and, thus, show up as artifacts. However, including multiples can potentially increase the illumination of the subsurface (Malcolm et al., 2009; Zuberi and Alkhalifah, 2014a, 2014b). Moreover, the enriched illumination provided by the wavepath of the multiple scattered energy also helps full-waveform inversion (FWI) retrieve low-wavenumber model updates (Alkhalifah and Wu, 2016; Berkhout and Verschuur et al, 2016; Guo and Alkhalifah, 2017; Wang et al., 2018; Alkhalifah et al., 2018; Alkhalifah and Guo, 2019).

So far, many studies have been devoted to imaging the Earth's surface-related multiples (Verschuur et al., 1992; Liu et al., 2011a), whereas for the more complex internal multiples, the solutions are limited (Malcolm et al., 2009). Recently, Marchenko type imaging algorithms have been developed, to retrieve the full subsurface Green's function (up- and down-going Green's function), that include all primaries and multiples based on the exact inverse-scattering theory (Behura et al., 2012; Wagenaar et al., 2014; Singh and Snieder, 2017). However, the Marchenko focusing method fails to reconstruct wavefields caused by vertical interfaces because 
the kinematic characteristics of up- and down-going Green's functions would not be similar with the vertical interface unless vertical seismic profile (VSP) data were used for additional control (Lomas et al., 2018). Singh and Curtis (2019) demonstrate a timereversed mirror imaging strategy to map the near-vertical structures using multiple scattered waves. Although these procedures only require a smooth reference model, imaging of multiples of different orders is not feasible.

Zuberi and Alkhalifah (2014) propose a three-step approach based on the interferometry that migrates any order of internal multiple separately, which is referred as generalized internal multiple imaging (GIMI). This approach transforms different order of internal multiples into the leading-order scattering term by performing additional correlations between the surface recorded data and its backpropagated wavefield at image points. It only relies on the background Green's function, often based on a smooth kinematically accurate version of the velocity model (Wang et al., 2017; Sun and Alkhalifah, 2018), similar to the velocities used for conventional migrations. The required number of interferometric cross-correlations is one less than the order of the term in the Born scattering series (or equal to the order of internal multiple) we intend to image. Then, the specific order of multiple can be imaged by a regular RTM procedure. Alkhalifah and Guo (2019) formulated the adjoint operation of the GIMI process and further used it to suppress the crosstalk artifacts resulting from the conventional GIMI using least-squares optimization. They also suggested using the leading-order term of the scattering series to extract wavepath updates for velocity 
model building. However, the additional computational cost of the interferometric step still hinders its application in large-scale cases (Wang et al., 2019).

In this paper, we implement the GIMI operation and its adjoint in the frequencydomain with significantly less computational cost of the interferometric step. This should benefit the entire process of the least-squares optimization of GIMI. Besides, we apply the energy norm imaging condition (Rocha et al., 2016; Sun and Alkhalifah, 2017) to isolate the reflectivity component of the image and the corresponding longwavelength energy extracted from the lower-order scattering. We start with introducing our frequency-domain least-squares GIMI theory. Then, we briefly review the different energy norm imaging types and present how to apply them in the least-squares GIMI. Finally, we show two synthetic examples of a salt flank model and a modified Marmousi model to verify the feasibility of the proposed strategy.

\section{THEORY}

\section{Frequency-Domain Least-Squares Generalized Internal Multiple Imaging Theory}

Our purpose for this section is to present the frequency-domain internal multiples imaging workflow in a least-squares scheme (including misfit function definition, gradient calculation, GIMI adjoint, and step length computation) and highlight its advantages compared to the time-domain implementation. We focus on imaging double-scattering energy here. 
Form the $l_{2}$ norm objective function $E$ as

$$
E(I)=\frac{1}{2}\left|R_{r s}^{-}-R_{r s}^{\mathrm{m}}(I)\right|_{2}^{2}=\frac{1}{2}\left|\Delta R_{r s}^{-}\right|_{2}^{2},
$$

where $I$ is the image obtained using the LS-GIMI, which corresponds to the reflectivity of second-order scattering and the long-wavelength wavepath energy from the primary (first-order) reflections, $R_{r s}^{-}$is the surface recorded data, $R_{r s}^{\mathrm{m}}(I)$ is the modeled reflections from the double-scattering image $I$ using the adjoint GIMI process, which is similar to Born modeling (de-migration) in the conventional LS-RTM, and $\Delta R_{r s}^{-}=R_{r s}^{-}-R_{r s}^{\mathrm{m}}(I)$.

As described by Zuberi and Alkhalifah (2014) and Alkhalifah and Guo (2019), the gradient compute of LS-GIMI includes the three following steps:

(1) Backpropagate the misfit data from the surface to the image domain to obtain the mainly upgoing wavefield (superscript ${ }^{-}$) from the source, $s$, to the image point, $x$, $G_{x_{S}}^{-}$. In mathematical terms, we can write this step as follows

$$
G_{x s}^{-}=G_{r x}^{b} \Delta R_{r s}^{-*}
$$

where $G_{r x}^{b}$ is the background Green's function from $x$ to $r$, and the $\Delta R_{r s}^{-*}$ is the complex conjugate (superscript ${ }^{*}$ ) of the misfit record. This step is same as the backpropagation process in LS-RTM or FWI.

(2) Then, we effectively transform the leading-order scattering term of the Born series to the wavepath term and the second-order to be the leading-order scattering term. This is achieved by using an interferometric cross-correlation of the backpropagated wavefield $G_{x s}^{-}$with the data residual $\Delta R_{r s}^{-}, G_{r x}^{-}=G_{x s}^{-*} \Delta R_{r s}^{-}$, which includes a summation over sources. In the time-domain, this step is a multidimensional cross- 
correlation operation with a computation complexity of $O\left(N^{2}\right)$, where $N$ represents the number of time samples. When it comes to the frequency-domain, the correlation degenerates into a multiplication for each frequency, which substantially reduces the computation cost. Therefore, the frequency-domain strategy allows for a more practical GIMI implementation. Also, to improve the accuracy of Green's function $G_{r x}^{-}$, we employ a frequency-domain deterministic deconvolution and supplement a proper damping, which is given by

$$
G_{r x}^{-}=G_{x s}^{-*} \Delta R_{r s}^{-} /\left(G_{x s}^{*} G_{x s}^{-}+\varepsilon\right),
$$

where $\varepsilon$ is a small positive real number.

(3) Finally, we invoke the zero-lag cross-correlation imaging condition to highlight the second-order scattering (first-order internal multiples) and formulate the gradient,

$$
\Delta I=\left(G_{x r}^{b}\right)^{+*} G_{r x}^{-}
$$

which includes a summation over receivers. The term $\left(G_{r x}^{b}\right)^{+}$corresponds to the background down-going (superscript ${ }^{+}$) wavefield. For the first iteration, $\Delta R_{r s}^{-}=R_{r s}^{-}$, equation 2-4 present the conventional GIMI, and in this case, the image is often contaminated by crosstalks from different order multiples.

To reduce the artifacts caused by the crosstalk and balance the wavepath energy in GIMI results, Alkhalifah and Guo (2019) formulate the adjoint GIMI for a least-squares implementation. As described in equation 2-4, the GIMI maps the data into a doublescattering image. Hence, the adjoint GIMI also follows three steps to model the full reflection data from image $I$. Unlike GIMI, now we move from image to data, so the steps given by Born modeling are as follows: 
(1) Isolating the upward scattered energy $G_{r x}^{-}$from the background Green's function $G_{r x}^{b+}$ with the principle of reciprocity

$$
G_{r x}^{-}=G_{r x}^{b+} I
$$

(2) Considering the image $I$ is the double-scattering reflectivity, a similar interferometric cross-correlation is needed for the Green's function $G_{r x}^{-}$

$$
G_{x s}^{-}=G_{r x}{ }^{*} R_{r s}^{-}
$$

(3) Finally, we propagate the interferometric result $G_{x s}^{-}$from subsurface point $x$ to receiver location $r$,

$$
R_{r s}^{\mathrm{m}}=G_{r x}^{b-} G_{x S}^{-}
$$

Until now, we can iteratively update the reflectivity image related to doublescattering as,

$$
I^{(i+1)}=I^{(i)}+\alpha \Delta I^{(i)}
$$

by minimizing the misfit function in equation 1 . We can update $I$ using the gradientor Newton-type optimization method (such as the steepest-descent, the conjugate gradient, or the L-BFGS methods), and $i$ represents the iteration index. In our application, we use the steepest-descent method, and the step length $\alpha$ is given by (Feng and Schuster, 2017),

$$
\alpha=\frac{\operatorname{real}\left[R_{r s}^{-*} * R_{r s}^{\mathrm{m}}\left(I^{(i)}\right)\right]}{\operatorname{real}\left[R_{r s}^{-*} * R_{r s}^{-}\right]} .
$$

Since this framework is with the bounds of linearized inversion, like LS-RTM, and does not update the velocity model, the background Green's function $G_{r x}^{b}$ remains stationary during the iterations. Furthermore, $G_{r x}^{b}$, given in the frequency-domain, is reasonably compact, and thus, can be often saved in computer memory; therefore, in 
the following iterations, the gradient and the GIMI adjoint calculation using equation $2,4,5$ and 7 are much faster than their time-domain equivalent.

\section{Energy Norm Imaging Conditions for LS-GIMI}

Next, we utilize the spatial gradients and time derivatives of wavefields to implement the energy norm imaging condition (Whitmore and Crawley, 2012; Rocha et al., 2016; Sun and Alkhalifah, 2017) in our LS-GIMI process to isolate the double-scattered reflectivity component from the GIMI image automatically. A spatial and temporal Fourier transform of the wavefield renders it a function of the wavenumber vector $\boldsymbol{k}$ $=\left[k_{x}, k_{y}, k_{z}\right]$ and the angular frequency $\omega$, in which we can apply the energy norm imaging condition in GIMI as,

$$
I_{E}=\mathbf{\square}\left(G_{x r}^{b}\right)^{+*} \cdot \mathbf{\square} G_{r x}^{-},
$$

where

$$
\begin{gathered}
\text { - } G_{r x}^{-}=\left(i v k_{x}, i v k_{y}, i v k_{z},-i \omega\right) G_{r x}^{-}, \\
\square\left(G_{r x}^{b}\right)^{+*}=\left(i v k_{x}, i v k_{y}, i v k_{z}, i \omega\right)\left(G_{r x}^{b}\right)^{+*} .
\end{gathered}
$$

Through further derivation, equation 10 leads to,

$$
I_{E}=\left[-\omega^{2} \cos 2 \theta+\omega^{2}\right]\left(G_{x r}^{b}\right)^{+*} G_{r x}^{-}=+2 \omega^{2} \sin ^{2} \theta\left(G_{x r}^{b}\right)^{+*} G_{r x}^{-} .
$$

In equation $13, \theta$ is the reflection angle and also the angle between the spatial gradient vectors, as shown in Figure 1a for the double-scattered reflection. We can apply a scaling factor to the second term in brackets, $I_{E}=\left[-\omega^{2} \cos 2 \theta+\omega^{2} \cos 2 \theta_{c}\right]\left(G_{x r}^{b}\right){ }^{+*} G_{r x}^{-}$, to control the contribution as a function of reflection angle $\theta_{c}$. To attenuate the low-wavenumber energy, which corresponds to having $\theta_{c}=90^{\circ}$, we set the scaling factor to -1 . Thus, the backscattering attenuation 
imaging condition is,

$$
I_{E}^{m i g}=\left[-\omega^{2} \cos 2 \theta-\omega^{2}\right]\left(G_{x r}^{b}\right)^{+*} G_{r x}^{-}=-2 \omega^{2} \cos ^{2} \theta\left(G_{x r}^{b}\right)^{+*} G_{r x}^{-} .
$$

Because these two imaging conditions (equations 13 and 14) are complementary with respect to a trigonometric function, the maximum contribution from one imaging condition is the minimum for the other one and vice versa. Therefore, we can image a narrow range of reflection angles near $90^{\circ}$, the tomographic term, by employing an exponential function associated with $I_{E}^{m i g}$ to $I_{E}$,

$$
I_{E}^{\text {tomo }}=I_{E} e^{-\alpha I_{E}^{\text {mig }}}
$$

where factor $\alpha>1$, and the $I_{E}^{m i g}$ is normalized, such that only the factor $\sin ^{2} \theta$ is accounted for in the exponential. In the example section, we use the symbols $I_{E}$ (equation 13), $I_{E}^{m i g}$ (equation 14) and $I_{E}^{\text {tomo }}$ (equation 15) to represent the conventional energy norm, backscattering attenuation, and the tomographic term imaging conditions, respectively.

We first test our frequency-domain GIMI by applying it to a simple salt flank-like model, as displayed in Figure 1a. The model consists of two homogeneous layers $(2400 \mathrm{~m} / \mathrm{s}$ and $2700 \mathrm{~m} / \mathrm{s})$, and a vertical reflector located on the left side of the model, which does not produce any primary reflections recorded in this limited-offset survey. Thus, the conventional RTM cannot image the vertical structure properly. The dimension of the model is $\mathrm{nx} * \mathrm{nz}=201 * 101$, and the grid interval is $15.24 \mathrm{~m}$ in both the horizontal and vertical directions. We employ 21 shots evenly distributed on the surface with a spacing of $152.4 \mathrm{~m}$. We generate synthetic data with frequencies 
ranging from $5 \mathrm{~Hz}$ to $25 \mathrm{~Hz}$ in a $2 \mathrm{~Hz}$ sampling interval using a unit Dirac source wavelet. Only the reflection data will be used for imaging; the migration model for the GIMI process is the first layer velocity $(2400 \mathrm{~m} / \mathrm{s})$.

Figure $1 \mathrm{~b}$ shows the result of the interferometric step for a receiver located at $\mathrm{x}=1524 \mathrm{~m}$. The first-order scattering term is propagating downward (the triangle area), which will produce the low-frequency wavepath components with the corresponding background downgoing wavefield $\left(G_{r x}^{b}\right)^{+}$in Figure 1c. On the other hand, the second-order scattering is heading in the upward direction (black arrows). Some of the energy in the second-order term is covered by the dominating first-order term, but it will not cause any interference because of the nearly opposite propagation directions, which will contribute to the second-order reflectivity image, as shown in Figure 2a. Figures $2 \mathrm{~b}$ and $2 \mathrm{c}$ show the two components of the image. Although the migration $I_{E}^{\text {mig }}$ and the tomographic $I_{E}^{\text {tomo }}$ term are well separated, we notice that $I_{E}^{m i g}$ have some vertical artifacts and low resolution. We intend to alleviate this issue by a LS-GIMI implantation, as we stated in the last section.

Note that the zero-scattering information $I_{E}^{\text {tomo }}$ in the first-order GIMI image fits the primary reflection in the adjoint GIMI when the $I_{E}^{m i} g_{\text {information generates double }}$ reflection (Alkhalifah and Guo, 2019). Hence in LS-GIMI with the energy norm imaging condition, the $I_{E}$ shown in Figure 2a, containing both tomographic and migration components, should be used for the adjoint GIMI to formulate the full modeled data $R_{r s}^{\mathrm{m}}$ to match the surface record $R_{r s}^{-}$. In this case, equation 5 is replaced 
by,

$$
G_{r x}^{-}=G_{r x}^{b+} I_{E}
$$

Next, we can update the $I_{E}$ and $I_{E}^{m i g}$ simultaneously by,

$$
\begin{aligned}
& I_{E}^{(i+1)}=I_{E}^{(i)}+\alpha \Delta I_{E}^{(i)}, \\
& I_{E}^{m i g(i+1)}=I_{E}^{m i g(i)}+\alpha \Delta I_{E}^{m i g(i)},
\end{aligned}
$$

The corresponding gradients for $I_{E}$ and $I_{E}^{m i g}$ are computed using equations 13 and 14 , respectively.

Figure 3 shows the LS-GIMI images for the salt flank like model after 20 iterations. Compared with the conventional GIMI images in Figure 2a, the LS-GIMI image $I_{E}$ (Figure 3a) exhibits a more focused double-scattering image and legible low-frequency wavepath energy between the subsurface reflectors and receiver locations (The triangle and quadrilateral area reflection wavepath come from the shallow and deep horizontal interface, respectively). Naturally, the image $I_{E}^{m i g}$ (Figure 3b) displays fewer vertical artifacts, better amplitude balancing, and higher resolution than those in Figure $2 \mathrm{~b}$. We also illustrate the convergence curve (Figure 3c) of the difference between the original surface data and the data from adjoint GIMI with the image $I_{E}$. The misfit decreases gradually to about 10 percent after ten iterations, which demonstrates that this objective function converges at a faster rate. For reference, the associated LS-RTM image of this flank model is shown in Figure 4. RTM images the horizontal interfaces, but the flank interface is missing because RTM does not have the ability to locate the multiple scattered energy with a constant background velocity model. 
All the GIMI images shown before are summed over sources and receivers. We also would like to share some intermediate results, which will help provide insights into GIMI. As a result, the GIMI images $I_{E}$ after the first and last iterations for a single source-receiver pair, whose ray path is plotted in Figure 1a, are displayed in Figures 5a and $5 \mathrm{~b}$, respectively. To facilitate the analysis, we overlay the schematic ray path on the GIMI images shown in Figure 5. According to Figure 5a, we can recognize the wellknown receiver-side "rabbit ear"-like sensitivity kernel for the first-order reflection (converted to wavepath thanks to GIMI) generated from the horizontal interface, and the intense wavepath energy covers the double-scattering reflectivity information. There are noticeable enhancements for the vertical interface imaging after the leastsquares update, as shown in Figure 5b. We also show the GIMI images, which include a summation over sources, for the same receiver location as Figure 5a. Note that the GIMI results after summation over the sources remarkably improve the resolution of the flank (Figure 5c), and the wavepath energy is well equalized after optimization (Figure 5d). In addition, with the reciprocity principle, the fan-shaped wavepath in Figure $5 \mathrm{~d}$ is consistent with the transmission kernel of a virtual source injected at the receiver location in Figure 1a. This investigation provides indisputable evidence that the first-order GIMI not only images the double-scattering, but also produces primary reflection kernels, which can be exploited to generate the low-wavenumber velocity update in reflection waveform inversion (Xu et al., 2012). For background updates, we need to make the data difference in equation 1 focus on the wavepath part of kernels. Reflection waveform inversion with GIMI is our next research topic. 


\section{A MARMOUSI MODEL EXAMPLE}

Now, we apply our frequency-domain LS-GIMI scheme to the more complicated, but modified, Marmousi model (Versteeg, 1994), which contains $\mathrm{nx} * \mathrm{nz}=251 * 101$ grids, shown in Figure 6a to illustrate its ability. The grid spacing in all directions is $10 \mathrm{~m}$. The model includes two "V" shaped reflectors (one side is given by faults), which is expected to produce double-scattering. In the Marmousi test, we use the same source wavelet and the same frequency range as the salt flank model. The acquisition geometry is still a full coverage of sources and receivers on the recording surface. In total, 126 sources are exploded at a depth of $30 \mathrm{~m}$ with a distance between adjacent sources of $20 \mathrm{~m}$. The wavefields are recorded at 251 receivers, covering the entire model for each shot, at the same depth with sources. The smoothed version of the Marmousi model in Figure $6 \mathrm{~b}$ is taken as the migration velocity model for GIMI.

We carry out the proposed LS-GIMI platform on the smoothed Marmousi model directly using the simulated data from the exact Marmousi model. Figure 7a demonstrates the first iteration result of LS-GIMI corresponding to the image $I_{E}$. Since our first-order GIMI is incorporated using the energy norm imaging condition, we can get a cleaner double-scattering image $I_{E}^{m i g}$, shown in Figure 7b, automatically without the application of a Laplacian filter. The LS-GIMI results after 30 iterations are shown in Figures $7 \mathrm{c}\left(I_{E}\right)$ and $7 \mathrm{~d}\left(I_{E}^{m i g}\right)$. Comparing the results in Figure 7, we can see that after applying our LS-GIMI scheme, the image $I_{E}^{m i g}$ shows a higher resolution and more focused double-scattering energy, and the wavepath energy illumination in the image 
$I_{E}$ is significantly improved as well. The final double-scattering reflectivity image precisely displays the two "V" shaped faults structure, where multiple reflections take place as we expected. Due to the limited frequency band, the LS-GIMI result cannot express the full wavenumber components of the accurate reflectivity model. To qualify the least-squares optimization, we show the steady descent of the misfit curve (Figure 8) and the adjoint record, which matches well with the original data (Figure 9). The least-squares image for the single-scattering is shown in Figure 10a. We sum Figure 10a and Figure $7 \mathrm{~d}$ to obtain a new image shown in Figure 10b, which is courtesy of the single and double-scattered reflections. As a result, the new image shows a certain improvement in the continuity of the event, especially for the right flank of the first "V", which is missing in the single-scattered least-squares image. The multiple crosstalk artifacts in the right side of the model have a slight increase due to the complex structures and the limited frequency slices, but it is acceptable to some extent.

\section{DISCUSSION}

To obtain the second-order scattering images, GIMI transfers the second-order scattering into the leading scattering term forming the migration component, and the primary scattering becomes the zero-order transmission wavepath energy between the subsurface point and the receiver location using an interferometric process. We deploy the energy norm imaging condition to separate the double-scattering reflectivity from the GIMI image. The main advantage of the energy norm imaging is that the amplitude 
and phase are accurately preserved. Compared with the low-cut filters (Zhang and Sun, 2009) or wavefield decomposition (Liu et al., 2011b) methods, the outputs here are more physically meaningful.

Unlike the time-domain implementation (Zubri and Alkhalifah, 2014; Alkhalifah and Guo, 2019), the frequency-domain GIMI provides more efficient interferometric operation and offers the possibility of higher-order GIMI. However, for a full-band image, the GIMI should be repeated for all frequencies, which is high in cost as well. Under the assumption that wavenumber redundancy exists in frequencies, applying the proposed LS-GIMI using a frequency sampling interval greater than the Nyquist limit can produce images with reasonable resolution. Furthermore, considering the doublescattering at small angles (a narrow range of scattering angles near $0^{\circ}$ ) cannot be produced in such a limited survey. It implies that the LS-GIMI needs an intense frequency sampling interval for imaging double-scattering to ensure the wavenumber continuity (Pratt, 1999; Sirgue and Pratt,2004). As stated earlier, except for the reflectivity image of double-scattering, another product of GIMI is the reflection wavepath. These wavepaths are beneficial for extracting the low-wavenumber components of the velocity model to avoid cycle-skipping in full-waveform inversion. Only one frequency can provide reasonable illumination for reflection waveform inversion, which is enriched with the help of multi-scattering energy (Alkhalifah and Wu, 2016; Alkhalifah, 2017). Therefore, the frequency-domain GIMI provided us with a new and efficient way to utilize the different order of reflections for inversion. 


\section{CONCLUSIONS}

We developed a frequency-domain least-squares generalized internal multiple imaging (LS-GIMI) schemes. In the frequency-domain, the interferometric step is evaluated by a deterministic deconvolution process, which provides an improved accuracy of function $G_{r x}$ and makes the proposed method reasonably efficient. We also apply the energy norm imaging conditions in LS-GIMI to image clean doublescattered reflections. The numerical examples demonstrate the effectiveness of the proposed method.

\section{ACKNOWLEDGEMENT}

We greatly appreciate the financial support of the National Key Research Development Program of China (2018YFA0702500-2018YFA0702504). We thank KAUST for its support and SWAG for the collaborative environment. Guanchao Wang also wishes to thank the China Scholarship Council for support to study abroad. 


\section{REFERENCES}

Alkhalifah, T., B. Sun, and Z. Wu, 2018, Full model wavenumber inversion: Identifying sources of information for the elusive middle model wavenumbers: Geophysics, 83, no. 6, R597-R610, https://doi.org/10.1190/geo2017-0775.1.

Alkhalifah, T., 2017, Sparse frequencies data inversion and the role of multi-scattered energy: 79th Annual International Meeting, EAGE, Expanded Abstracts, 1-5, http://dx.doi.org/10.3997/2214-4609.201700507.

Alkhalifah, T., and Z. Wu, 2016, Multiscattering inversion for low-model wavenumbers: Geophysics, $\quad 81, \quad$ no. $\quad 6, \quad$ R417-R428, https://doi.org/10.1190/geo2015-0650.1.

Alkhalifah, T., and Q. Guo, 2019, Subsurface wavefields based on the generalized internal multiple imaging: Geophysical Journal International, 219, no. 2, 12124324, https://doi.org/10.1093/gii/ggz340.

Behura, J., K. Wapenaar, and R. Snieder, 2012, Newton-Marchenko-Rose Imaging: 82nd Annual International Meeting, SEG, Expanded Abstracts, 1-6, https://doi.org/10.1190/segam2012-1531.1.

Berkhout, A. J., and D. J. Verschuur, 2016, Enriched seismic imaging by using multiple scattering: The Leading Edge, 35, no. 2, 128-133, https://doi.org/10.1190/tle35020128.1.

Claerbout, J. F., 1971, Toward a unified theory of reflector mapping: Geophysics, 36, no. 3, 467-481, https://doi.org/10.1190/1.1440185. 
Feng, Z., and G. T. Schuster, 2017, Elastic least-squares reverse time migration: Geophysics, 82, no. 2, S143-S157, https://doi.org/10.1190/geo2016-0254.1.

Guo, Q., and T. Alkhalifah, 2017, Elastic reflection based waveform inversion with a nonlinear approach: Geophysics, 82, no. 6, R309-R321, https://doi.org/10.1190/geo2016-0407.1.

Liu, Y., X. Chang, D. Jin, R. He, H. Sun, and Y. Zheng, 2011a, Reverse time migration of multiples for subsalt imaging: Geophysics, 76, no. 5, WB209-WB216, https://doi.org/10.1190/geo2010-0312.1.

Liu, F., G. Zhang, S. A. Morton, and J. P. Leveille, 2011b, An effective imaging condition for reverse-time migration using wavefield decomposition: Geophysics, 76, no. 1, S29 - S39, https://doi.org/10.1190/1.3533914.

Lomas, A., S. Singh, and A. Curtis, 2018, Marchenko imaging of both vertical and horizontal interfaces using VSP data: 88th Annual International Meeting, SEG, Expanded Abstracts, 5027-5031, https://doi.org/10.1190/segam2018-2996742.1.

Malcolm, A. E., B. Ursin, and M. V. De Hoop, 2009, Seismic imaging and illumination with internal multiples: Geophysical Journal International, 176, no. 3, 847-864, https://doi.org/10.1111/j.1365-246X.2008.03992.x.

Pratt, R. G., 1999, Seismic waveform inversion in the frequency domain, Part 1: Theory and verification in a physical scale model: Geophysics, 64, no. 3, 888-901, https://doi.org/10.1190/1.1444597.

Rocha, D., N. Tanushev, and P. Sava, 2016, Acoustic wavefield imaging using the energy norm: Geophysics, 81, no. 4, S151-S163, https://doi.org/10.1190/geo2015- 


\section{1.}

Singh, S., and R. Snieder, 2017, Strategies for imaging with Marchenko-retrieved Green's functions: Geophysics, 82, no. 4, Q23-Q37, https://doi.org/10.1190/geo2016-0293.1.

Singh, S., and A. Curtis, 2019, Imaging vertical interfaces using acoustic timereversal: Geophysics, 84, no. 3, Q1-Q11, https://doi.org/10.1190/geo2018-0135.1.

Sirgue, L., and R. G. Pratt, 2004, Efficient waveform inversion and imaging: A strategy for selecting temporal frequencies: Geophysics, 69, no. 1, 231-248, https://doi.org/10.1190/1.1649391.

Sun, B. B., and T. Alkhalifah, 2017, Wavefield separation by energy norm Born scattering: 87th Annual International Conference and Exhibition, SEG, Expanded Abstracts, 1481-1485, https://doi.org/10.1190/segam2017-17557895.1.

Sun, B. B., and T. Alkhalifah, 2018, Automatic wave-equation migration velocity analysis by focusing subsurface virtual sources: Geophysics, 83, no. 2, U1-U8, https://doi.org/10.1190/geo2017-0213.1.

Verschuur, D. J., A. J. Berkhout, and C. P. A. Wapenaar, 1992, Adaptive surface-related multiple elimination: Geophysics, 57, no. 9, 1166-1177, https://doi.org/10.1190/1.1443330.

Versteeg, R., 1994, The Marmousi experience: Velocity model determination on a synthetic complex data set: The Leading Edge, 13, no. 9, 927-936, https://doi.org/10.1190/1.1437051.

Wang, G. C., S. Wang, Q. Du, and S. Yuan, 2017, Traveltime-based reflection full- 
waveform inversion for elastic medium: Journal of Applied Geophysics, 141, 6876, https://doi.org/10.1016/j.jappgeo.2017.04.009.

Wang, G. C., S. Wang, J. Song, C. Dong, and M. Zhang, 2018, Elastic reflection traveltime inversion with decoupled wave equation: Geophysics, 83, no. 5, R463R474, https://doi.org/10.1190/geo2017-0631.1.

Wang, G. C., T. Alkhalifah, Q. Guo, and S. Wang, 2019, Frequency Domain Generalized Internal Multiple Imaging with Energy norm: 81st Annual International Meeting, EAGE, Expanded Abstracts, 1-5, https://doi.org/10.3997/2214-4609.201901020.

Wapenaar, K., J. Thorbecke, J. van der Neut, F. Broggini, E. Slob, and R. Snieder, 2014, Marchenko imaging: Geophysics, 79, no. 3, WA39-WA57, https://doi.org/10.1190/geo2013-0302.1.

Whitmore, N. D., and S. Crawley., 2012, Applications of RTM inverse scattering imaging conditions: 82nd Annual International Meeting, SEG, Expanded Abstracts, 1-6, https://doi.org/10.1190/segam2012-0779.1.

Xu, S., D. Wang, F. Chen, G. Lambare, and Y. Zhang, 2012, Inversion on reflected seismic wave: 82nd Annual International Meeting, SEG, Expanded Abstracts, 17, https://doi.org/10.1190/segam2012-1473.1.

Zhang, Y., and J. Sun, 2009, Practical issues of reverse time migration: True amplitude gathers, noise removal and harmonicsource encoding: International Geophysical Conference and Exposition, 204-204, https://doi.org/10.1190/1.3603729.

Zuberi, M. A. H., and T. Alkhalifah, 2014a, Generalized internal multiple imaging 
(GIMI) using feynman-like diagrams: Geophysical Journal International, 197, no.

3, 1582-1592, https://doi.org/10.1093/gji/ggt527.

Zuberi, M. A. H., and T. Alkhalifah, 2014b, Generalized internal multiple imaging:

Geophysics, 79, no. 5, S207-S216, https://doi.org/10.1190/geo2013-0287.1. 


\section{FIGURE CAPTIONS}

Figure 1 (a) The Salt flank-like velocity model with a first-order reflection ray path reflected by the horizontal interface (in solid black line) and a double-bounce ray path corresponding to the vertical interface (in dashed black line); the functions $G_{r x}^{-}$(b) and $\left(G_{r x}^{b}\right)^{+}$(c) for a frequency of $15 \mathrm{~Hz}$ at location $\mathrm{x}=1524 \mathrm{~m}$.

Figure 2 (a) The image $I_{E}$; (b) the image $I_{E}^{m i g}$ and (c) the image $I_{E}^{\text {tomo }}$.

Figure 3 (a) The least-squares GIMI image $I_{E}$ after 20 iterations, and (b) the corresponding image $I_{E}^{m i g}$; (c) The convergence curve of the objective function.

Figure 4 The least-squares RTM image after 20 iterations.

Figure 5 Deep analysis of the GIMI products. (a) The GIMI image $I_{E}$ of a single source-receiver pair corresponding to the ray path shown in Figure 1a (after the first iteration of the least-squares optimization); (b) The updated version of (a); (c) The GIMI image $I_{E}$ of the same receiver location with (a), but summation over sources (after the first iteration of the least-squares optimization); (d) The updated version of (c).

Figure 6 The modified Marmousi model (a) used to simulate recorded data. (b) The smoothed version of (a) used for LS-GIMI.

Figure 7 The LS-GIMI images corresponding to (a) $I_{E}$ and (b) $I_{E}^{m i g}$ after the first iteration of the least-squares optimization; The final LS-GIMI images (c) $I_{E}$ and (d) $I_{E}^{m i g}$ after 30 iterations. The intense energy in the image $I_{E}$ is caused by the large impedance contrast at the top of the Marmousi model. 
Figure 8 The convergence curve of the objective function for the Marmousi model

Figure 9 The real part of the original data (red lines) by the true model and the adjoint data (blue lines) by the image in Figure $7 \mathrm{c}$ at $15 \mathrm{~Hz}$ (a) and $19 \mathrm{~Hz}$ (b), for $64^{\text {th }}$ shot gather.

Figure 10 (a) The LS-RTM image of the Marmousi model, (b) the sum of the LSRTM and LS-GIMI images 


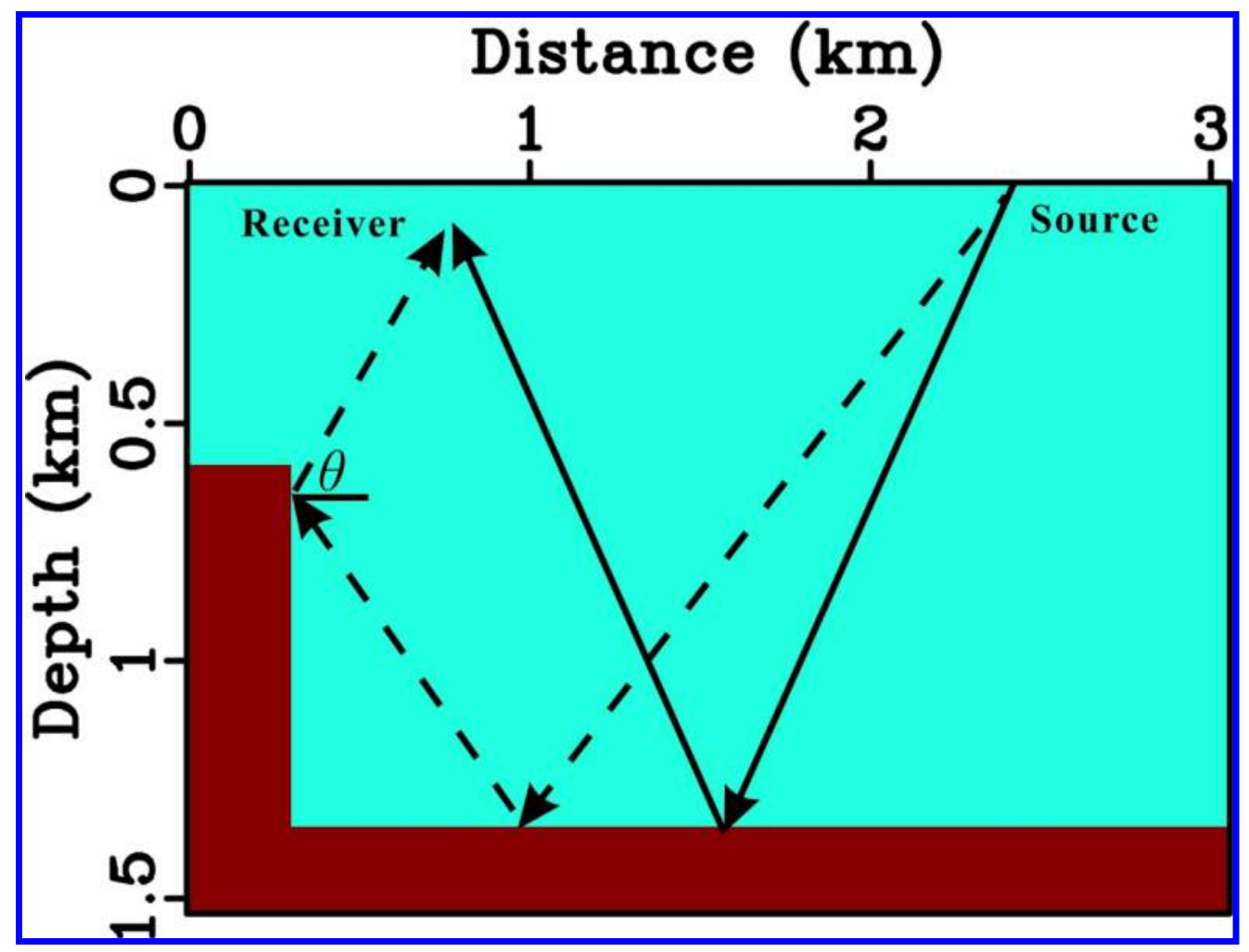

Figure number and letter: Figure 1a. Figure 1 (a) The Salt flank-like velocity model with a first-order reflection ray path reflected by the horizontal interface (in solid black line) and a double-bounce ray path corresponding to the vertical interface (in dashed black line); the functions $\mathrm{G}_{\mathrm{rx}}{ }^{-}(\mathrm{b})$ and $\left(\mathrm{G}_{\mathrm{rx}}{ }^{\mathrm{b}}\right)^{+}$(c) for a frequency of $15 \mathrm{~Hz}$ at location $\mathrm{x}=1524 \mathrm{~m}$.

$189 \times 143 \mathrm{~mm}(300 \times 300 \mathrm{DPI})$ 


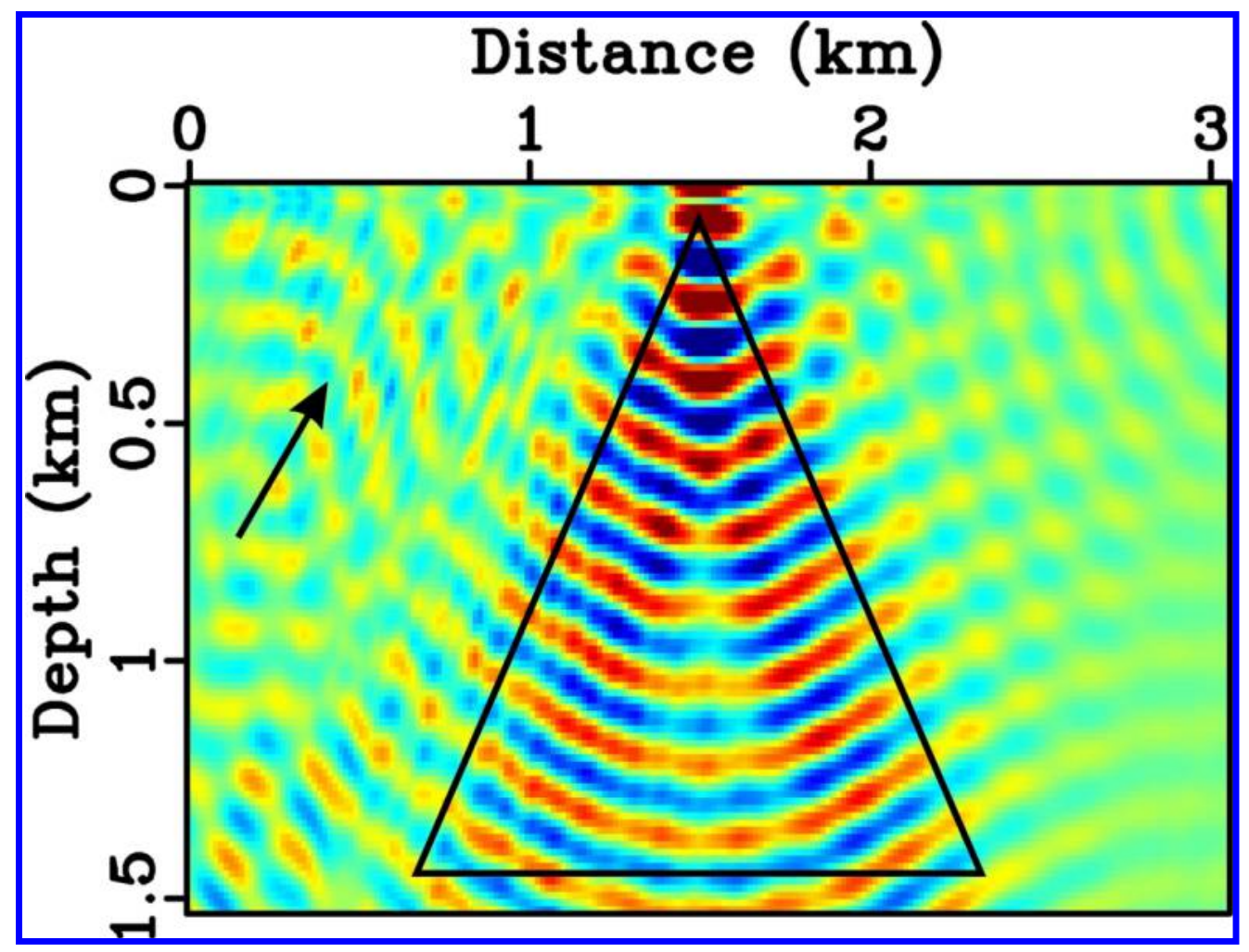

Figure number and letter: Figure 1b. Figure 1 (a) The Salt flank-like velocity model with a first-order reflection ray path reflected by the horizontal interface (in solid black line) and a double-bounce ray path corresponding to the vertical interface (in dashed black line); the functions $\mathrm{Grx}_{r{ }^{-}}$(b) and $\left(\mathrm{G}_{\mathrm{rx}}{ }^{\mathrm{b}}\right)^{+}$(c) for a frequency of $15 \mathrm{~Hz}$ at location $\mathrm{x}=1524 \mathrm{~m}$.

$189 \times 143 \mathrm{~mm}(300 \times 300 \mathrm{DPI})$ 

.

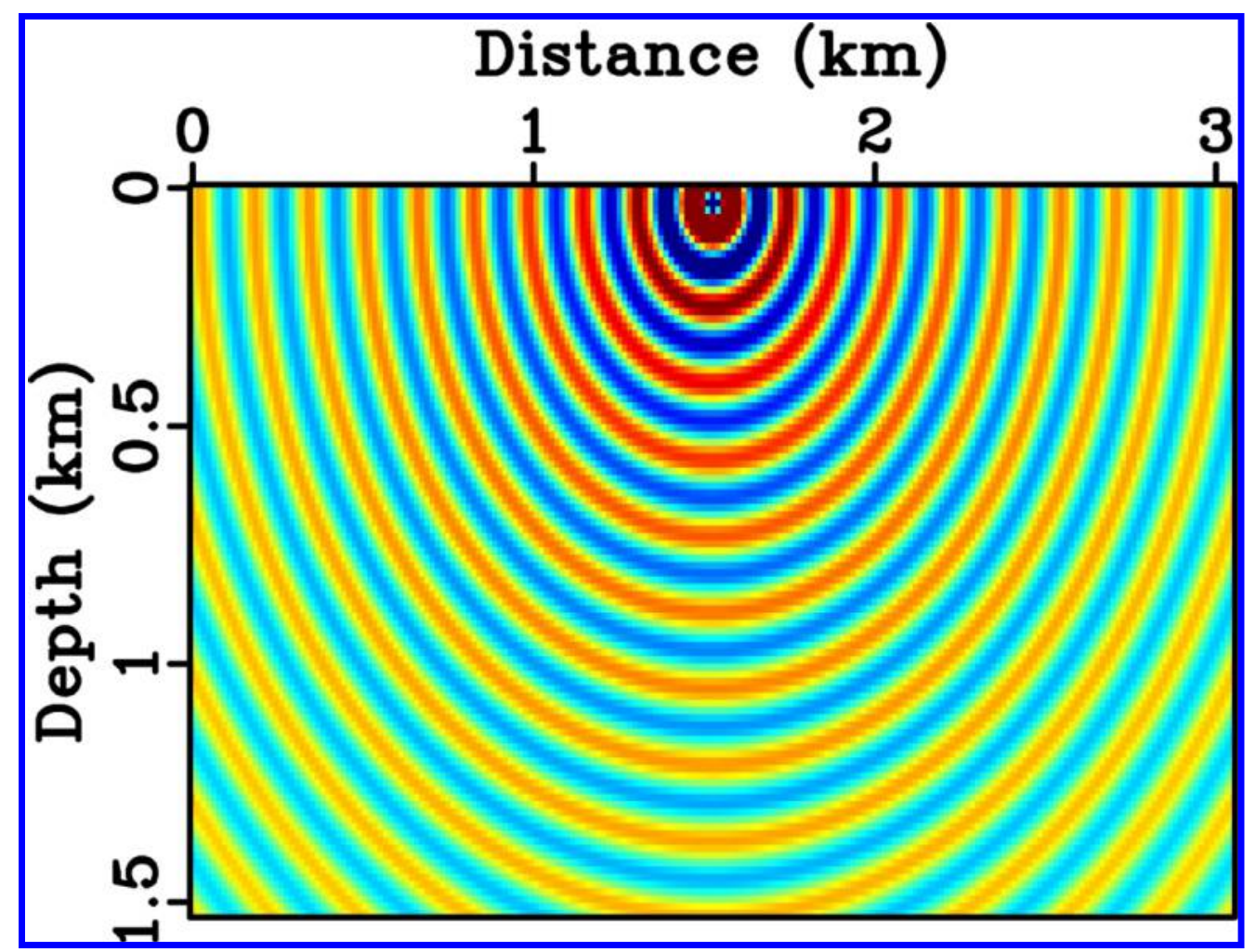

Figure number and letter: Figure 1c. Figure 1 (a) The Salt flank-like velocity model with a first-order reflection ray path reflected by the horizontal interface (in solid black line) and a double-bounce ray path corresponding to the vertical interface (in dashed black line); the functions $\mathrm{G}_{\mathrm{rx}^{-}}{ }^{-}(\mathrm{b})$ and $\left(\mathrm{G}_{\mathrm{rx}}{ }^{\mathrm{b}}\right)^{+}$(c) for a frequency of $15 \mathrm{~Hz}$ at location $\mathrm{x}=1524 \mathrm{~m}$.

$189 \times 143 \mathrm{~mm}(300 \times 300 \mathrm{DPI})$ 


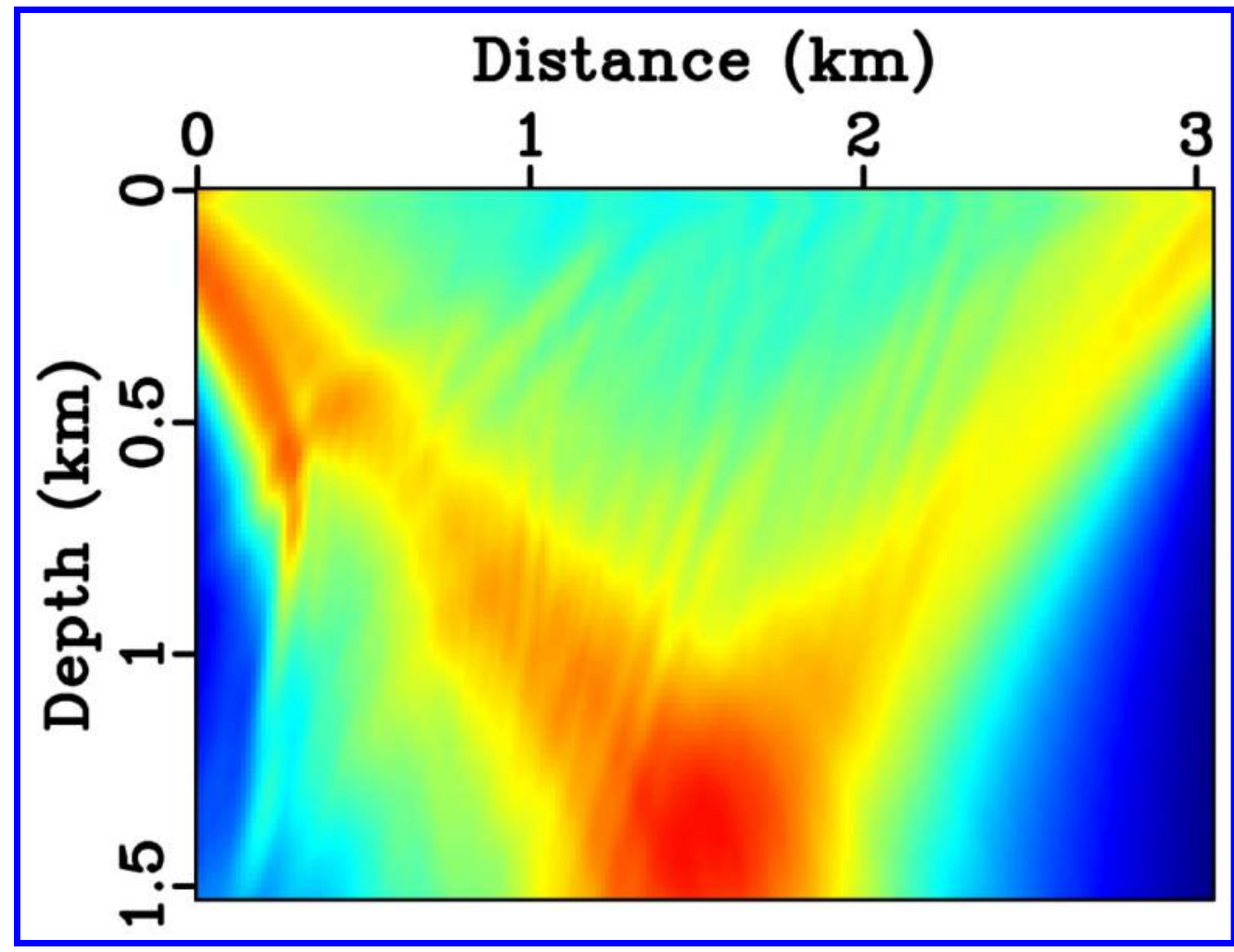

Figure number and letter: Figure 2a. Figure 2 (a) The image $\mathrm{I}_{\mathrm{E}}$; (b) the image $\mathrm{I}_{\mathrm{E}}^{\mathrm{mig}}$ and (c) the image $\mathrm{I}_{\mathrm{E}}^{\text {tomo }}$. $193 \times 147 \mathrm{~mm}(300 \times 300 \mathrm{DPI})$ 


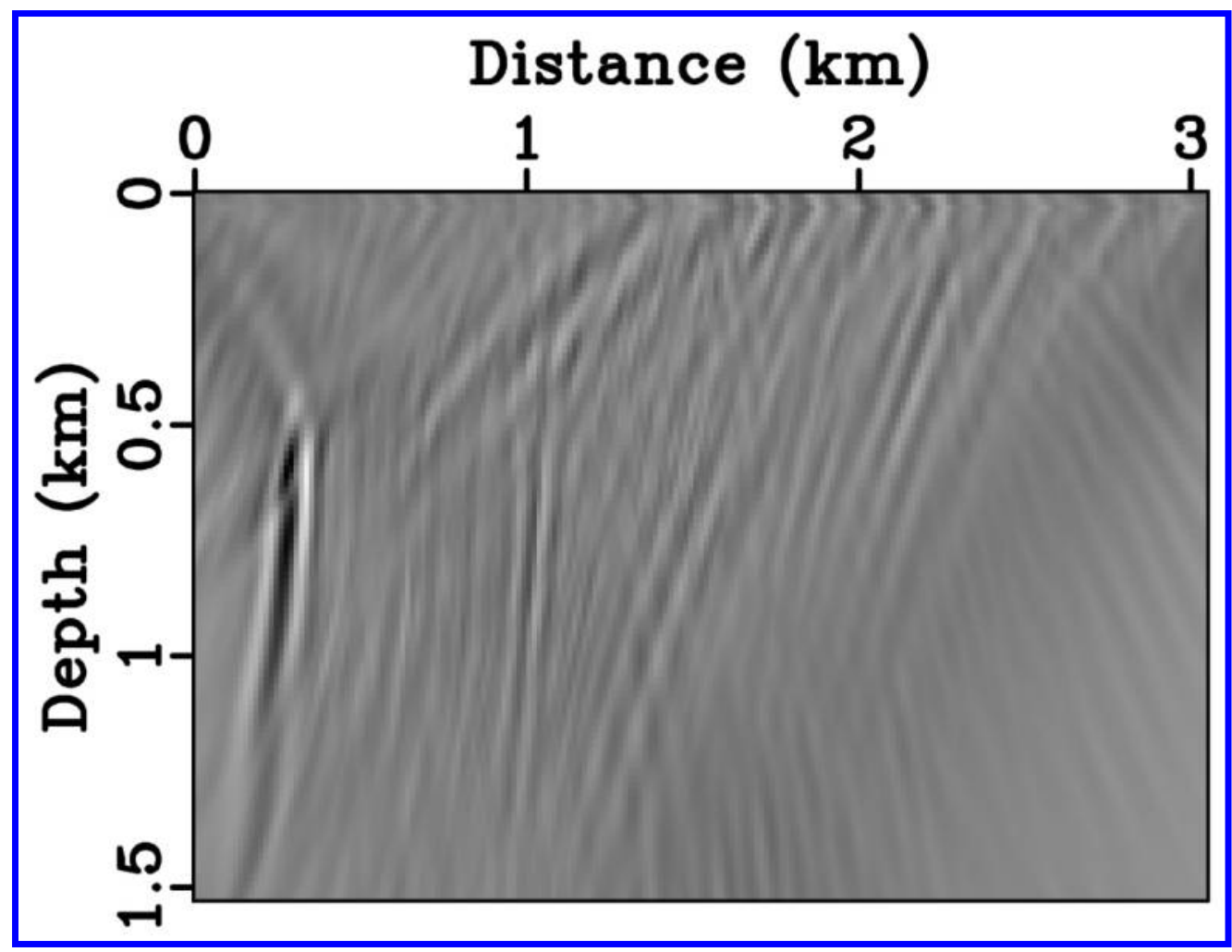

Figure number and letter: Figure $2 b$. Figure 2 (a) The image $\mathrm{I}_{\mathrm{E}} ;(\mathrm{b})$ the image $\mathrm{I}_{\mathrm{E}}{ }^{\mathrm{mig}}$ and $(c)$ the image $\mathrm{I}_{\mathrm{E}}^{\text {tomo }}$.

$193 \times 147 \mathrm{~mm}(300 \times 300 \mathrm{DPI})$ 


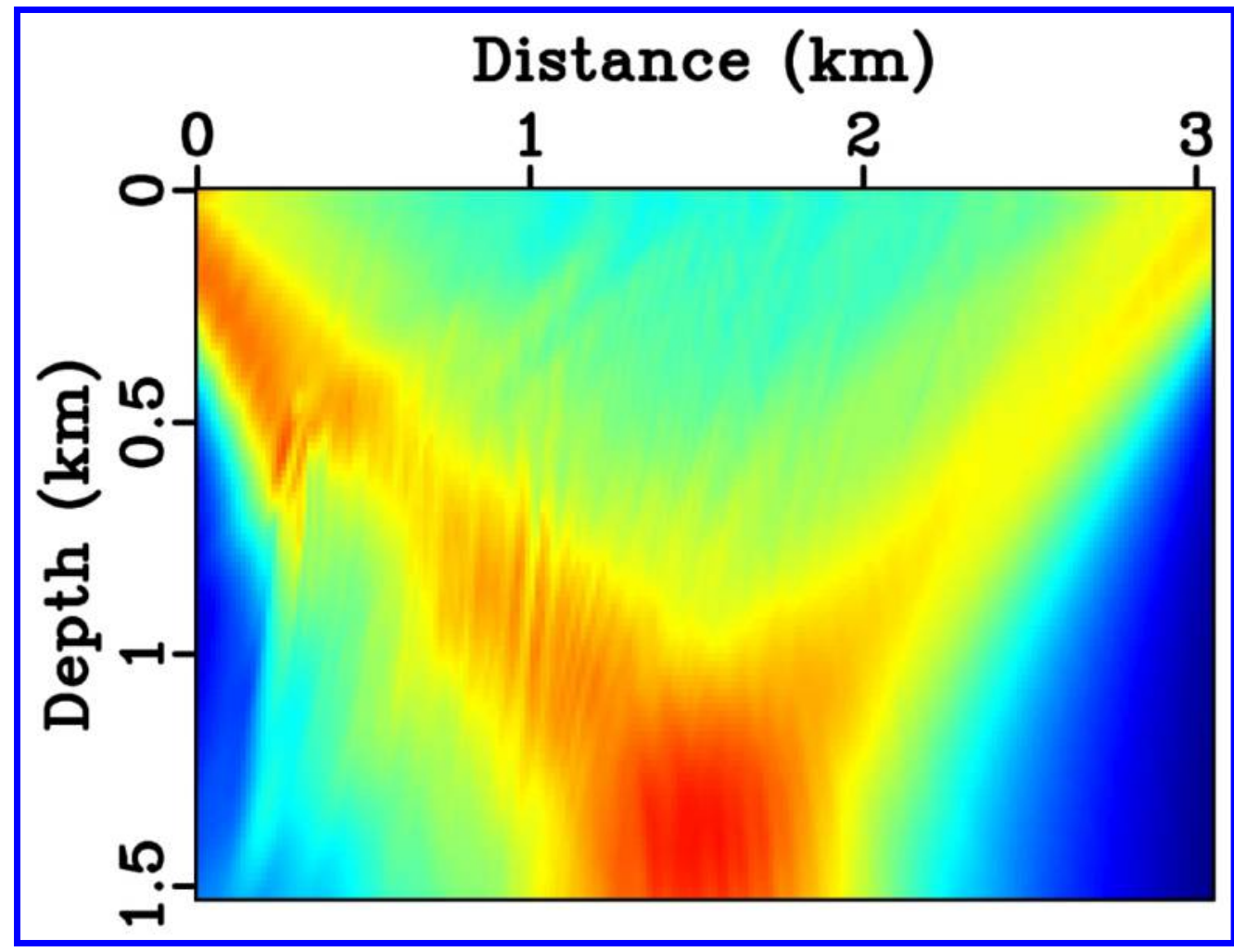

Figure number and letter: Figure $2 c$. Figure 2 (a) The image $\mathrm{I}_{\mathrm{E}} ;(\mathrm{b})$ the image $\mathrm{I}_{\mathrm{E}}^{\mathrm{mig}}$ and (c) the image $\mathrm{I}_{\mathrm{E}}^{\text {tomo }}$. $193 \times 147 \mathrm{~mm}(300 \times 300 \mathrm{DPI})$ 


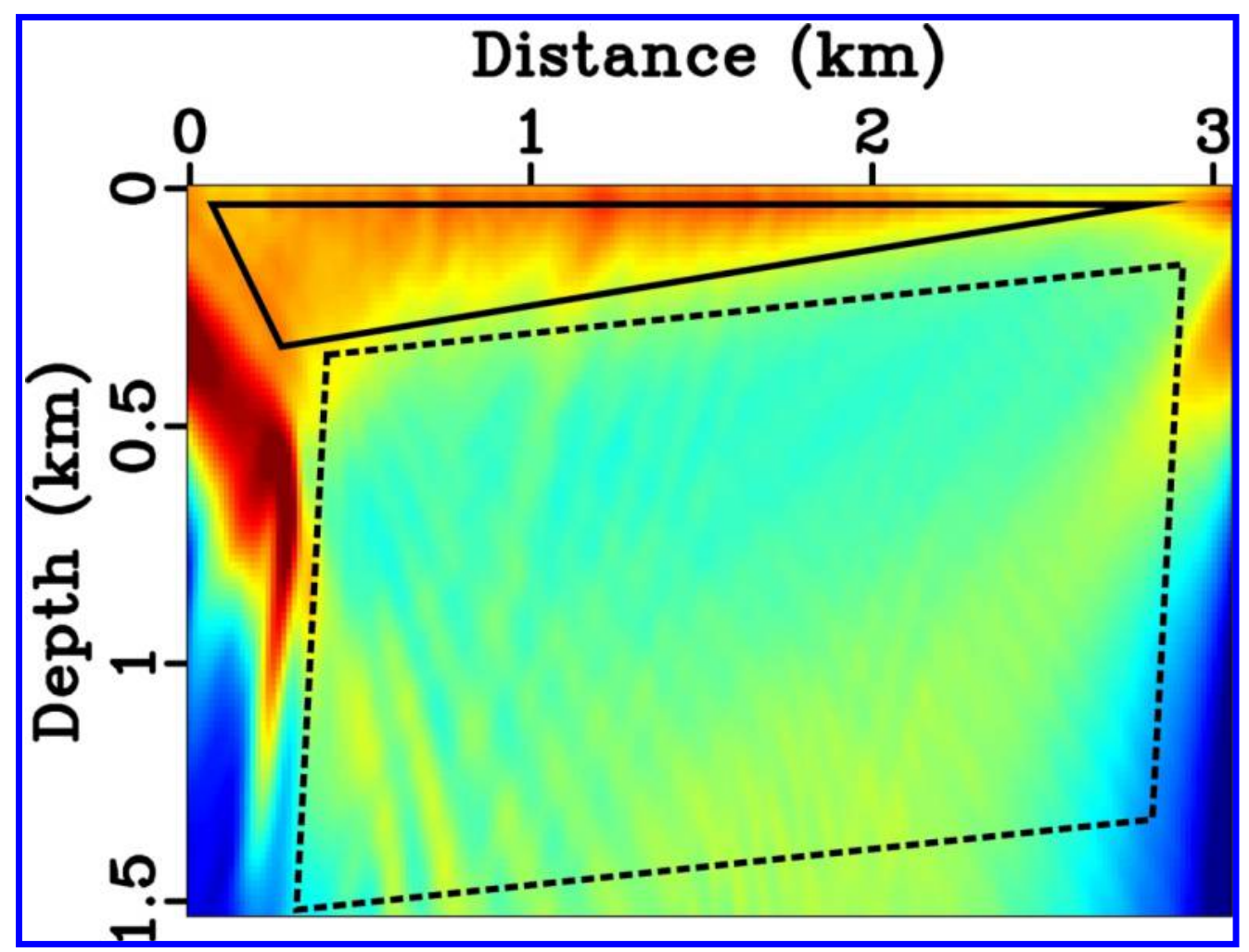

Figure number and letter: Figure 3a. Figure 3 (a) The least-squares GIMI image $\mathrm{I}_{\mathrm{E}}$ after 20 iterations, and (b) the corresponding image $\mathrm{I}_{\mathrm{E}}^{\mathrm{mig}}$; (c) The convergence curve of the objective function. $188 \times 143 \mathrm{~mm}(300 \times 300$ DPI) 


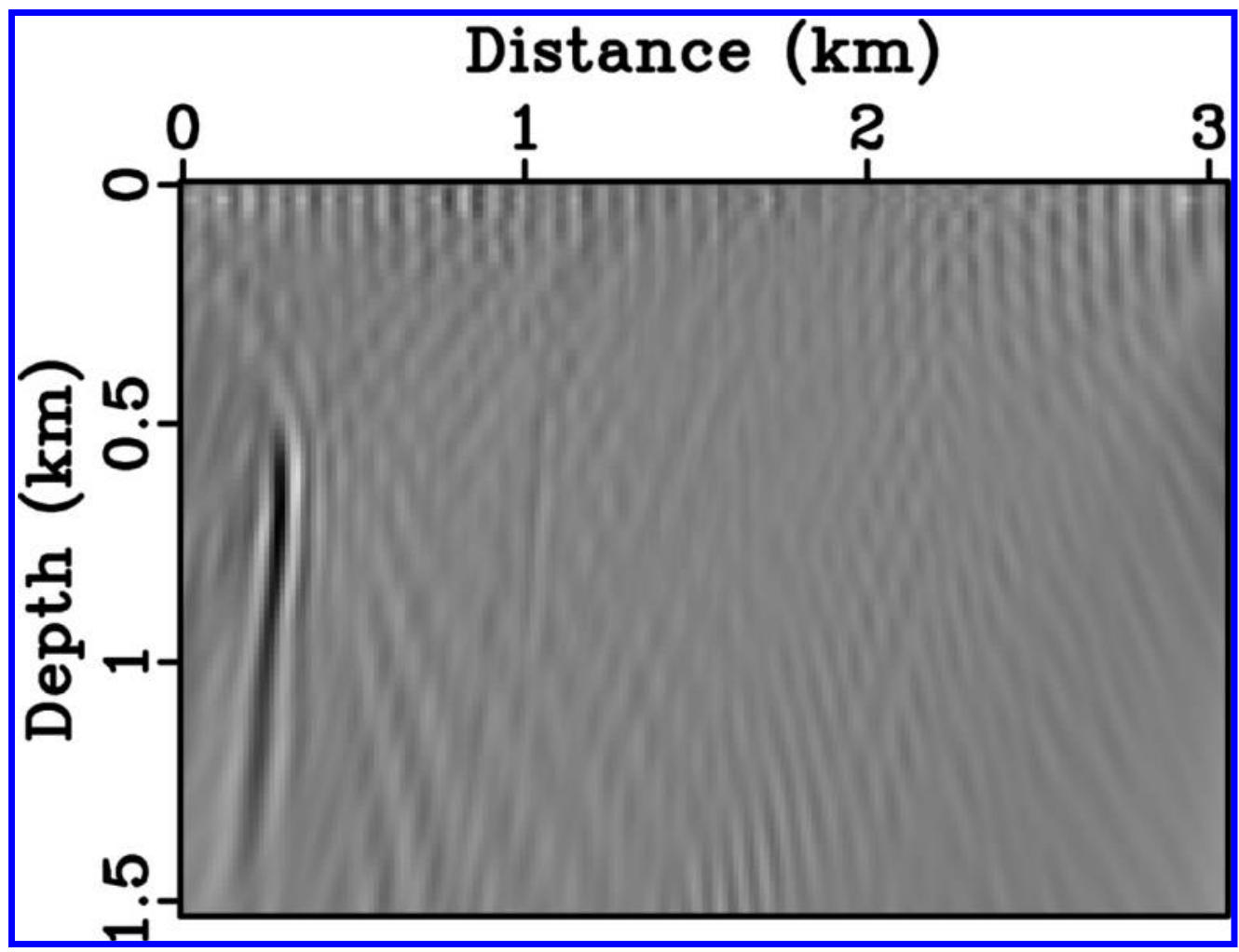

Figure number and letter: Figure 3b. Figure 3 (a) The least-squares GIMI image $\mathrm{I}_{\mathrm{E}}$ after 20 iterations, and (b) the corresponding image $\mathrm{I}_{\mathrm{E}}^{\mathrm{mig}}$; (c) The convergence curve of the objective function. $189 \times 143 \mathrm{~mm}(300 \times 300 \mathrm{DPI})$ 


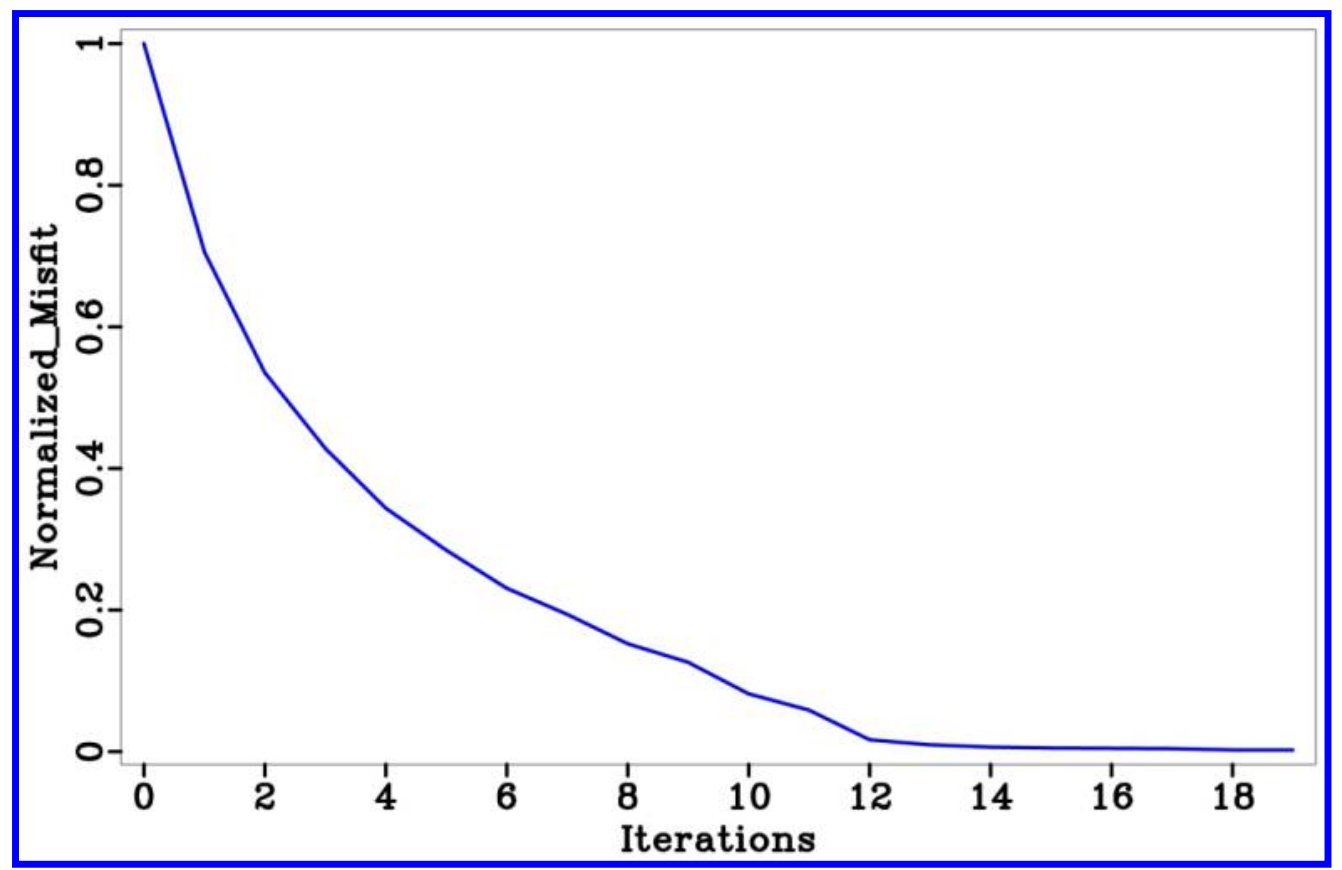

Figure number and letter: Figure 3c. Figure 3 (a) The least-squares GIMI image $\mathrm{I}_{\mathrm{E}}$ after 20 iterations, and (b) the corresponding image $\mathrm{I}_{\mathrm{E}}^{\mathrm{mig}}$; (c) The convergence curve of the objective function. $345 \times 223 \mathrm{~mm}(300 \times 300 \mathrm{DPI})$ 


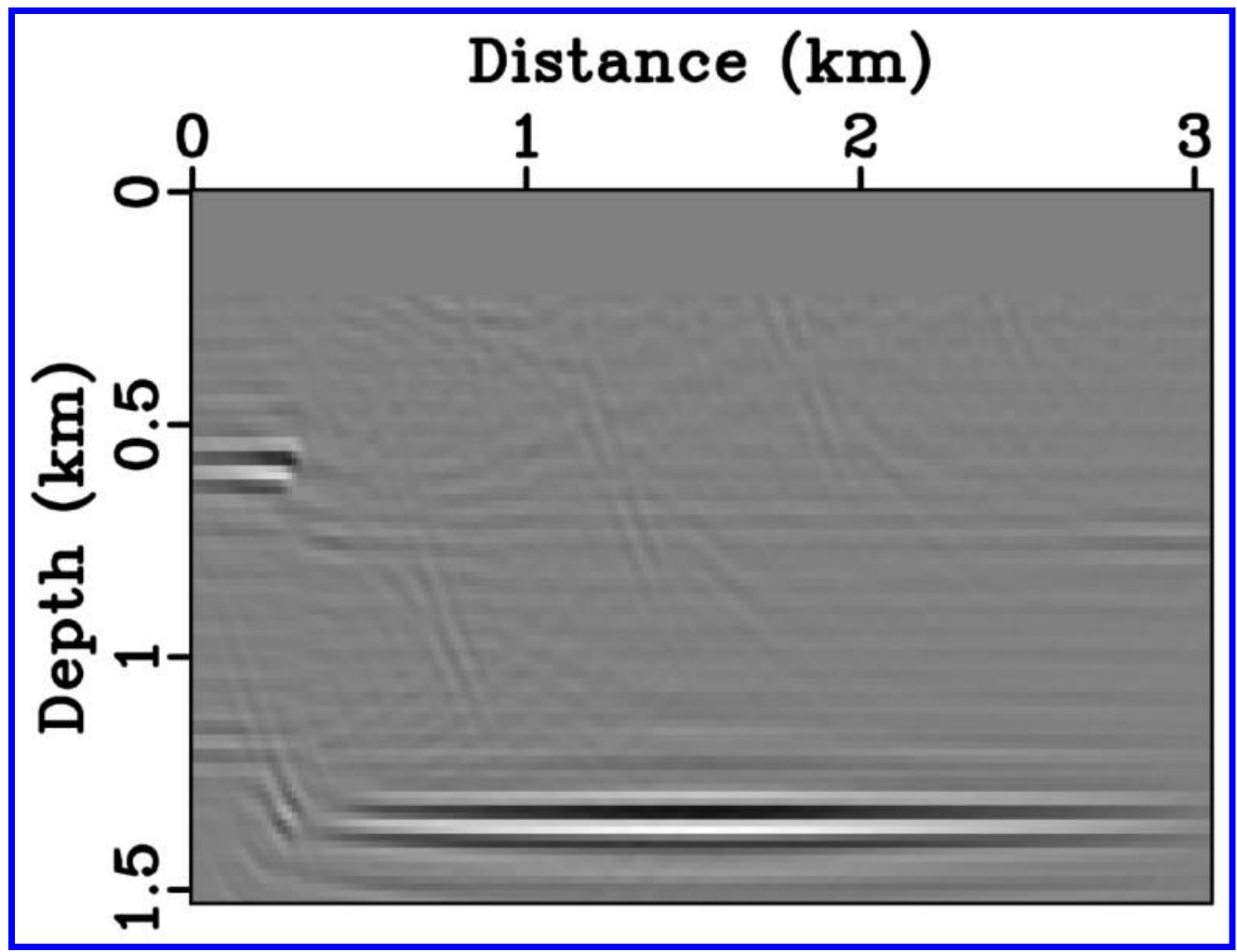

Figure number and letter: Figure 4. Figure 4 The least-squares RTM image after 20 iterations. $193 \times 147 \mathrm{~mm}(300 \times 300 \mathrm{DPI})$ 


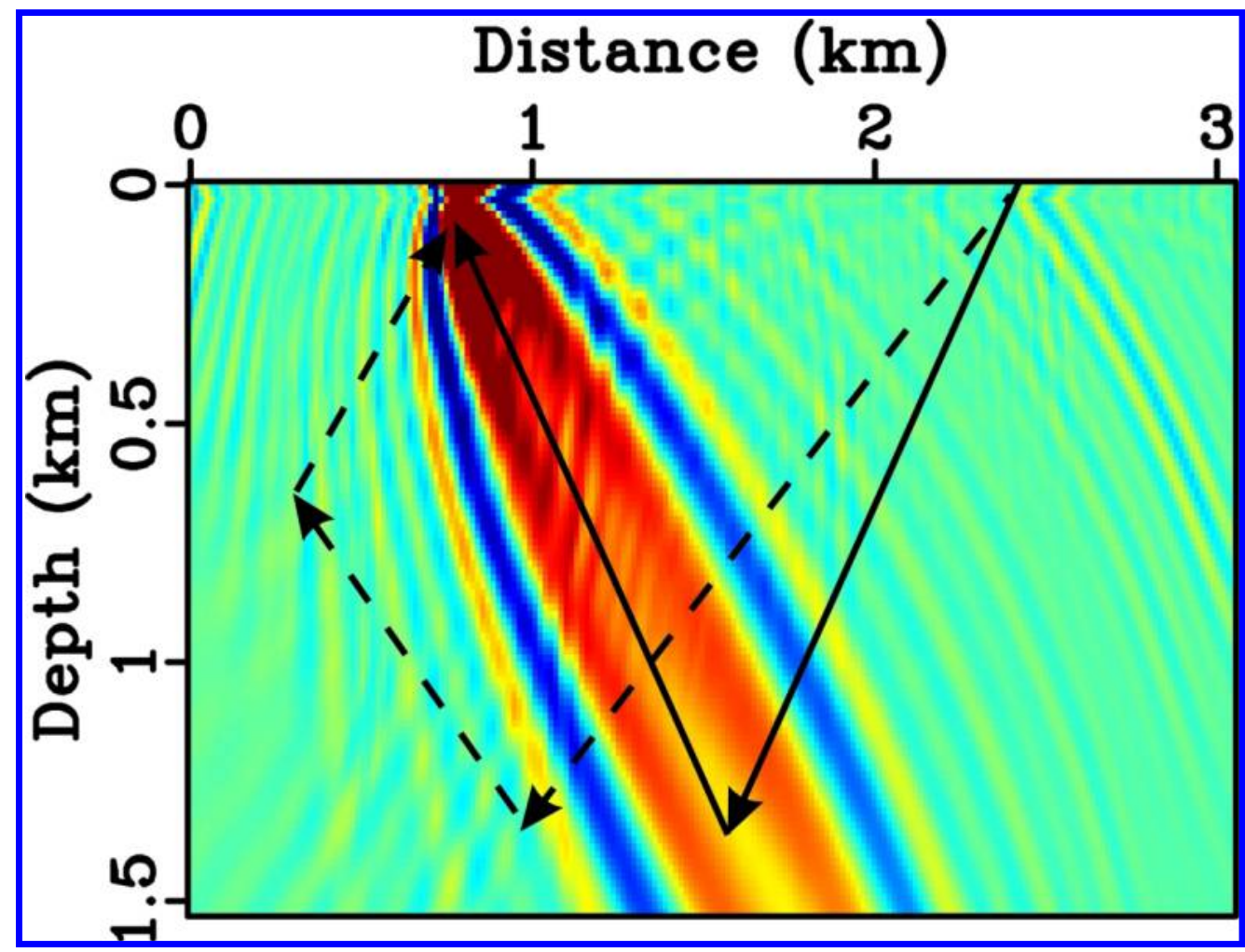

Figure number and letter: Figure 5a. Figure 5 Deep analysis of the GIMI products. (a) The GIMI image $\mathrm{I}_{\mathrm{E}}$ of a single source-receiver pair corresponding to the ray path shown in Figure 1a (after the first iteration of the least-squares optimization); (b) The updated version of (a); (c) The GIMI image $I_{E}$ of the same receiver location with (a), but summation over sources (after the first iteration of the least-squares optimization); (d) The updated version of (c).

$189 \times 143 \mathrm{~mm}(300 \times 300 \mathrm{DPI})$ 


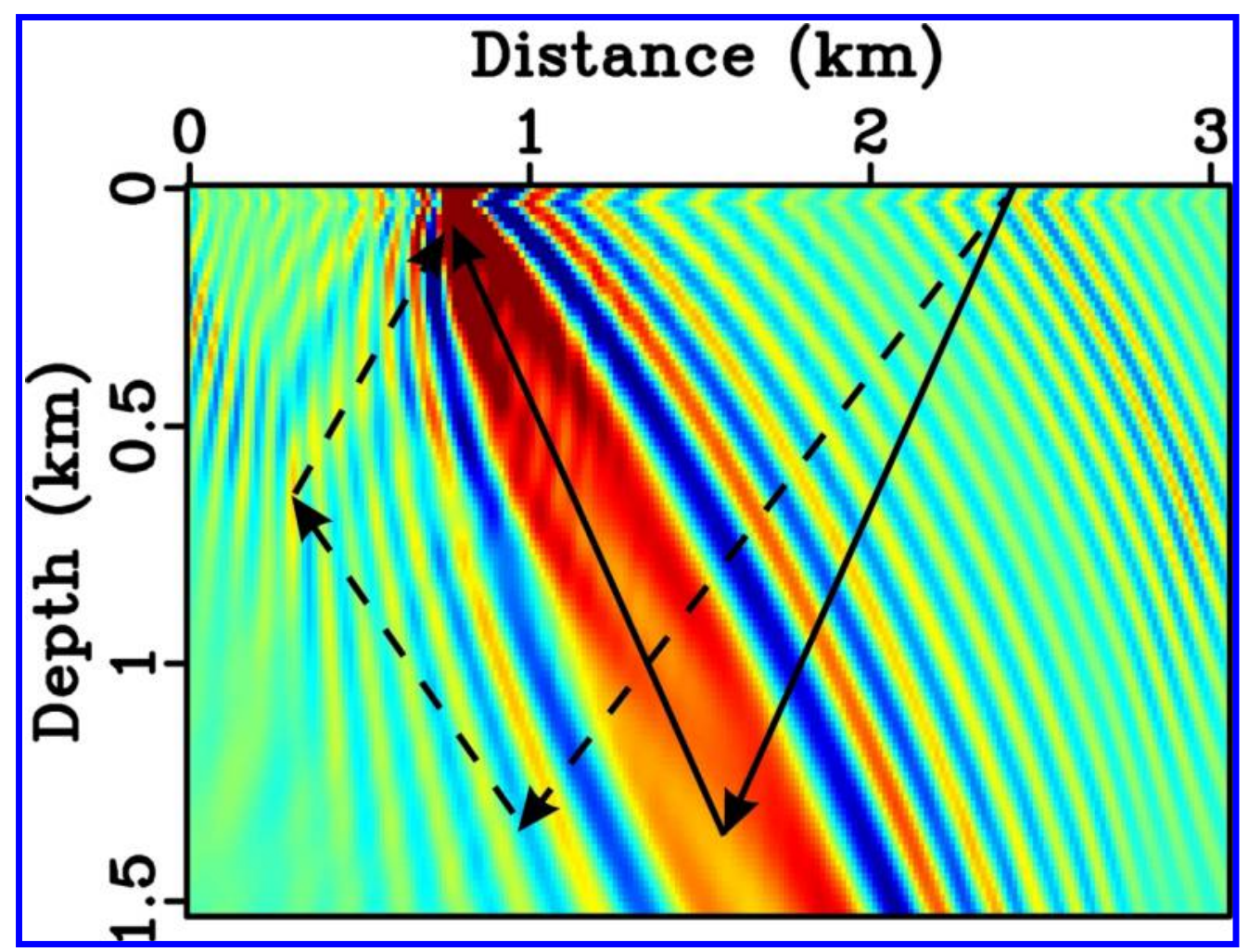

Figure number and letter: Figure 5b. Figure 5 Deep analysis of the GIMI products. (a) The GIMI image $I_{E}$ of a single source-receiver pair corresponding to the ray path shown in Figure 1a (after the first iteration of the least-squares optimization); (b) The updated version of (a); (c) The GIMI image $I_{E}$ of the same receiver location with (a), but summation over sources (after the first iteration of the least-squares optimization); (d) The updated version of (c).

$189 \times 143 \mathrm{~mm}(300 \times 300 \mathrm{DPI})$ 


(20)

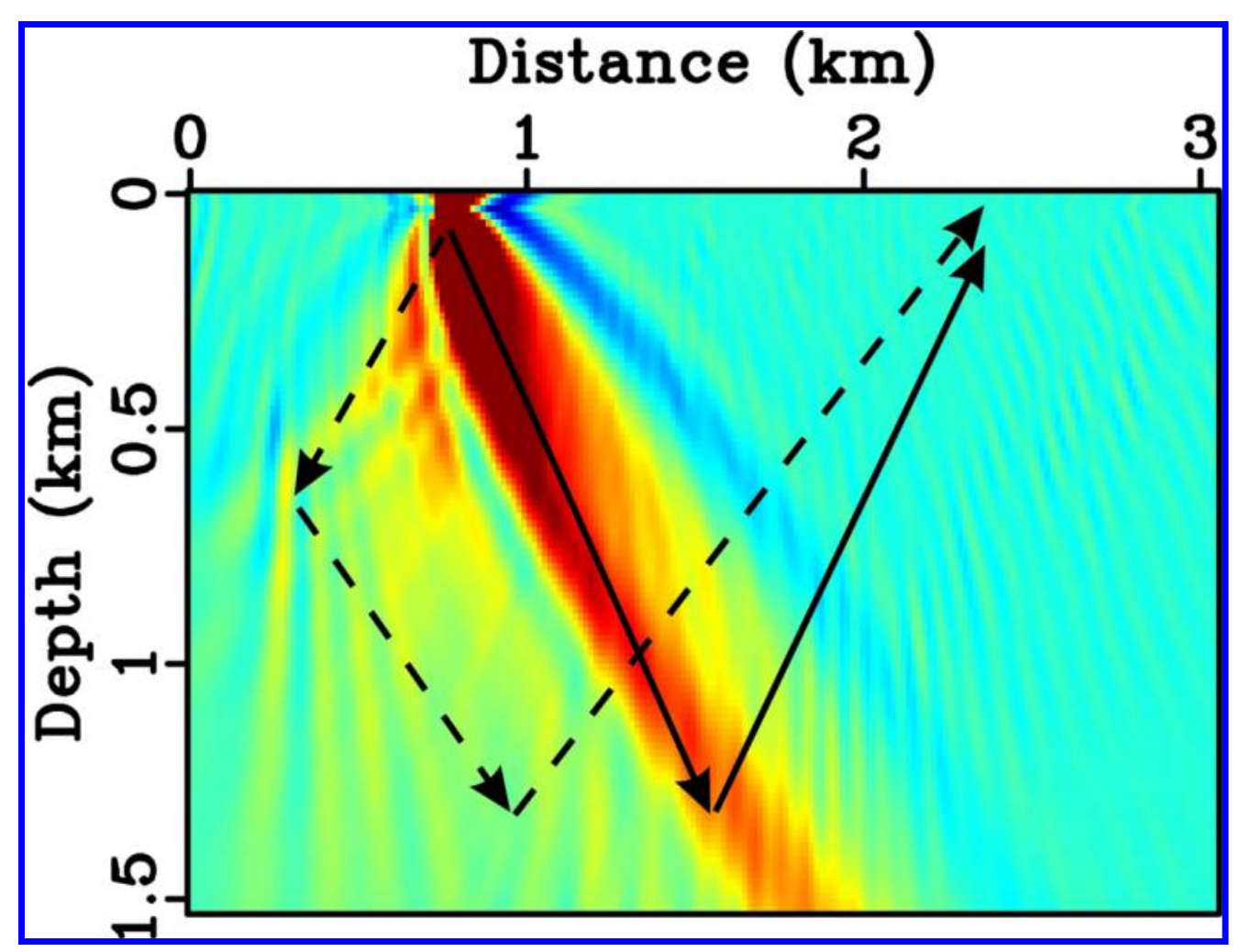

Figure number and letter: Figure 5c. Figure 5 Deep analysis of the GIMI products. (a) The GIMI image $I_{E}$ of a single source-receiver pair corresponding to the ray path shown in Figure 1a (after the first iteration of the least-squares optimization); (b) The updated version of (a); (c) The GIMI image $\mathrm{I}_{E}$ of the same receiver location with (a), but summation over sources (after the first iteration of the least-squares optimization); (d) The updated version of (c).

$189 \times 143 \mathrm{~mm}(300 \times 300 \mathrm{DPI})$ 


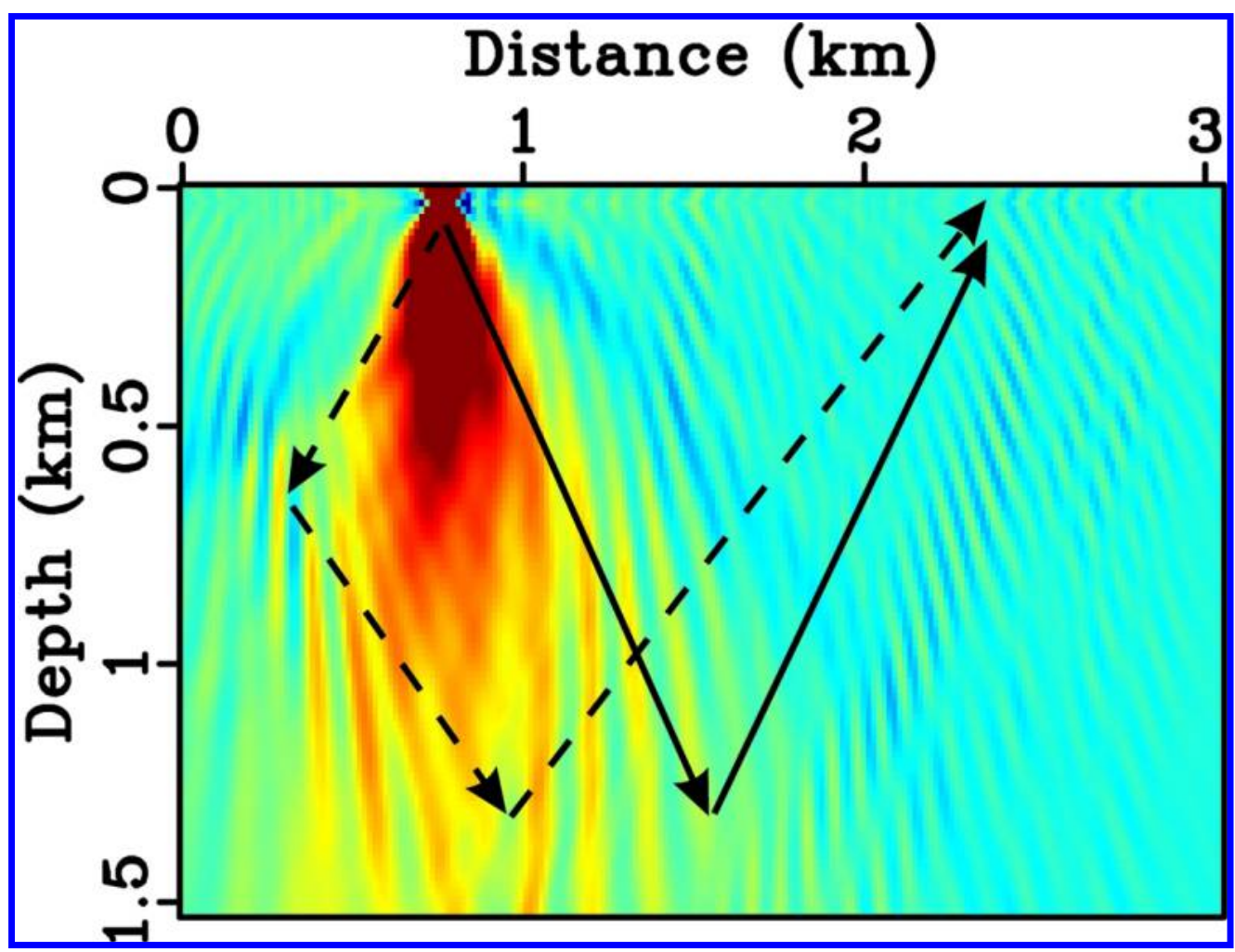

Figure number and letter: Figure 5d. Figure 5 Deep analysis of the GIMI products. (a) The GIMI image $\mathrm{I}_{\mathrm{E}}$ of a single source-receiver pair corresponding to the ray path shown in Figure 1a (after the first iteration of the least-squares optimization); (b) The updated version of (a); (c) The GIMI image $I_{E}$ of the same receiver location with (a), but summation over sources (after the first iteration of the least-squares optimization); (d) The updated version of (c).

$189 \times 143 \mathrm{~mm}(300 \times 300 \mathrm{DPI})$ 


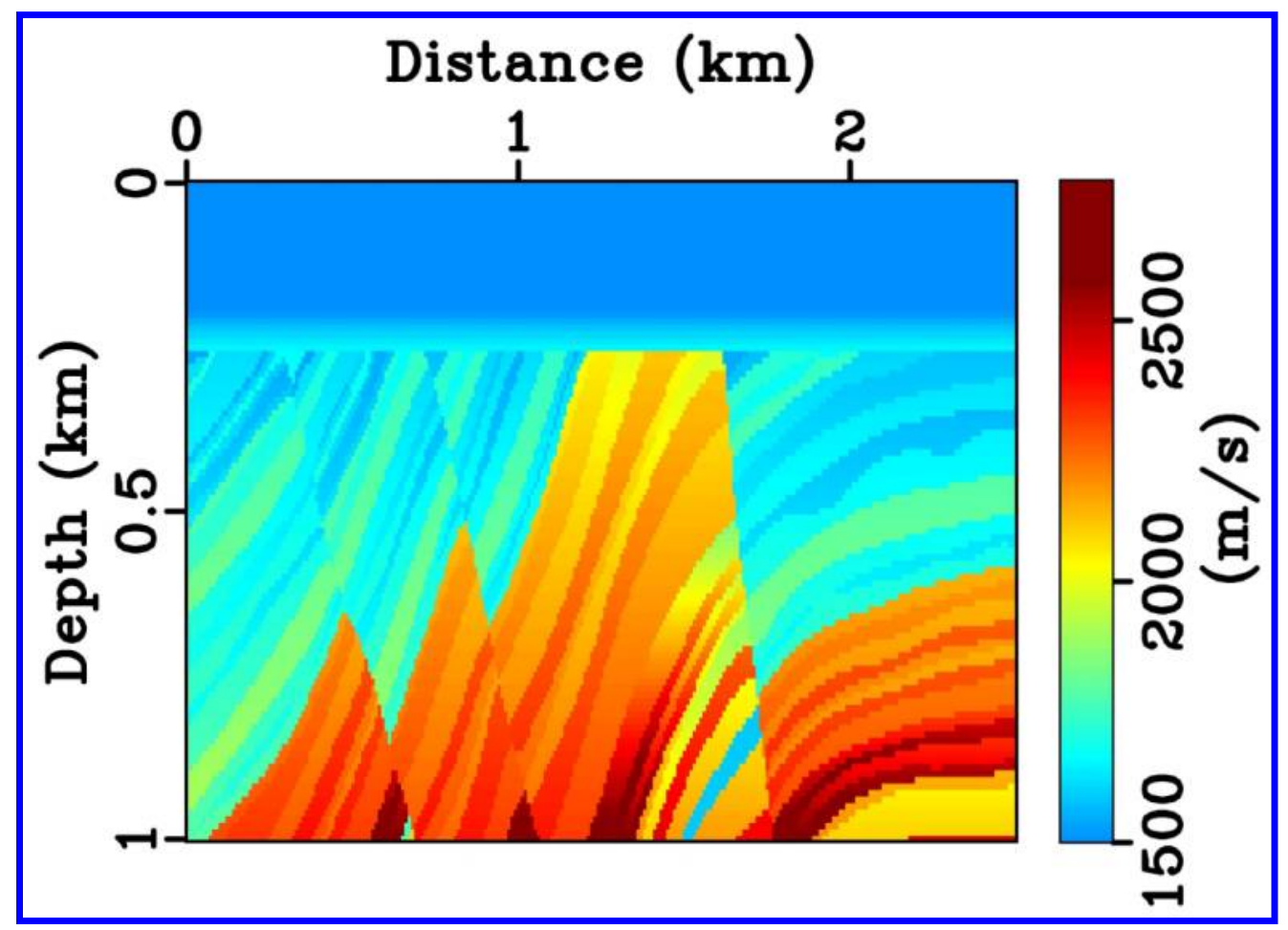

Figure number and letter: Figure 6a. Figure 6 The modified Marmousi model (a) used to simulate recorded data. (b) The smoothed version of (a) used for LS-GIMI.

$214 \times 154 \mathrm{~mm}(300 \times 300 \mathrm{DPI})$ 


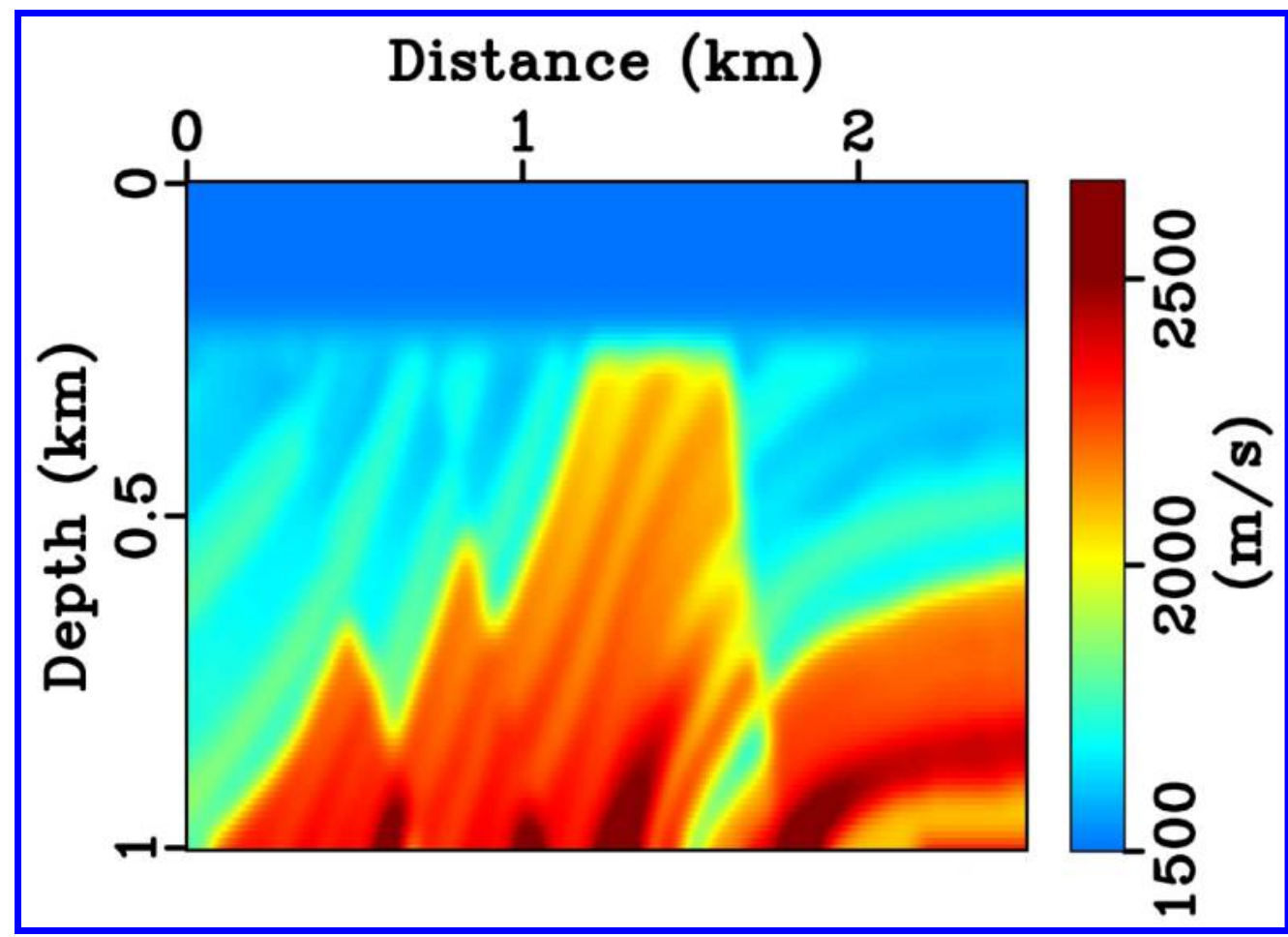

Figure number and letter: Figure 6b. Figure 6 The modified Marmousi model (a) used to simulate recorded data. (b) The smoothed version of (a) used for LS-GIMI.

$214 \times 154 \mathrm{~mm}(300 \times 300 \mathrm{DPI})$ 
Figure number and letter: Figure 7a. Figure 7 The LS-GIMI images corresponding to (a) $I_{E}$ and (b) $I_{E}$ mig after the first iteration of the least-squares optimization; The final LS-GIMI images (c) $I_{E}$ and (d) $I_{E}{ }^{\text {mig }}$ after 30 iterations. The intense energy in the image $\mathrm{I}_{\mathrm{E}}$ is caused by the large impedance contrast at the top of the Marmousi model.

$$
199 \times 144 \mathrm{~mm}(300 \times 300 \mathrm{DPI})
$$




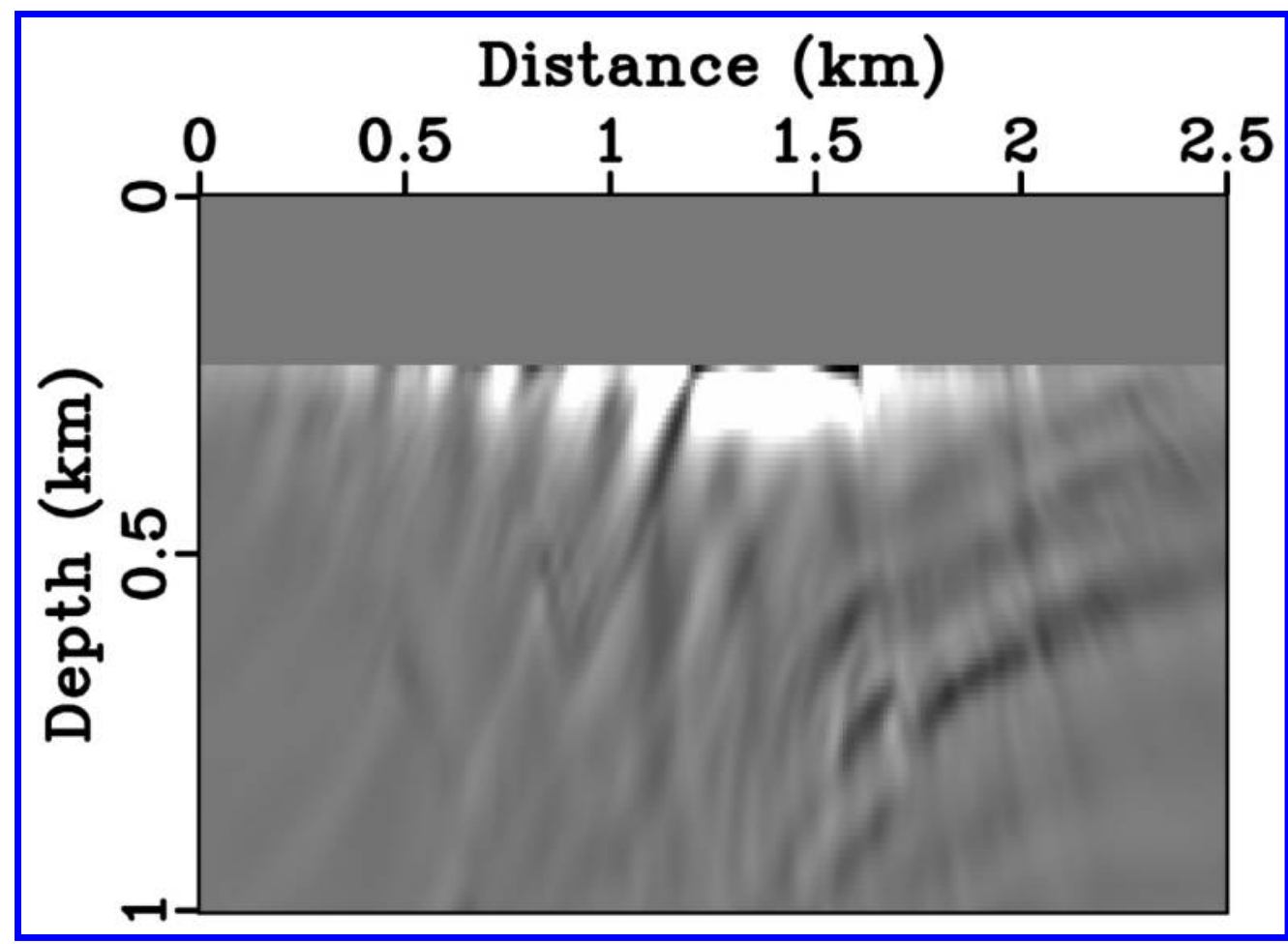

Figure number and letter: Figure 7b. Figure 7 The LS-GIMI images corresponding to (a) $\mathrm{I}_{E}$ and (b) $\mathrm{I}_{E}{ }^{\mathrm{mig}}$ after the first iteration of the least-squares optimization; The final LS-GIMI images (c) $I_{E}$ and (d) $I_{E}{ }^{m i g}$ after 30 iterations. The intense energy in the image $\mathrm{I}_{E}$ is caused by the large impedance contrast at the top of the Marmousi model.

$199 \times 144 \mathrm{~mm}(300 \times 300 \mathrm{DPI})$ 
Figure number and letter: Figure 7c. Figure 7 The LS-GIMI images corresponding to (a) $I_{E}$ and (b) $I_{E}{ }^{m i g}$ after the first iteration of the least-squares optimization; The final LS-GIMI images (c) $I_{E}$ and (d) $I_{E}{ }^{m i g}$ after 30 iterations. The intense energy in the image $\mathrm{I}_{E}$ is caused by the large impedance contrast at the top of the Marmousi model.

$$
199 \times 144 \mathrm{~mm}(300 \times 300 \text { DPI })
$$




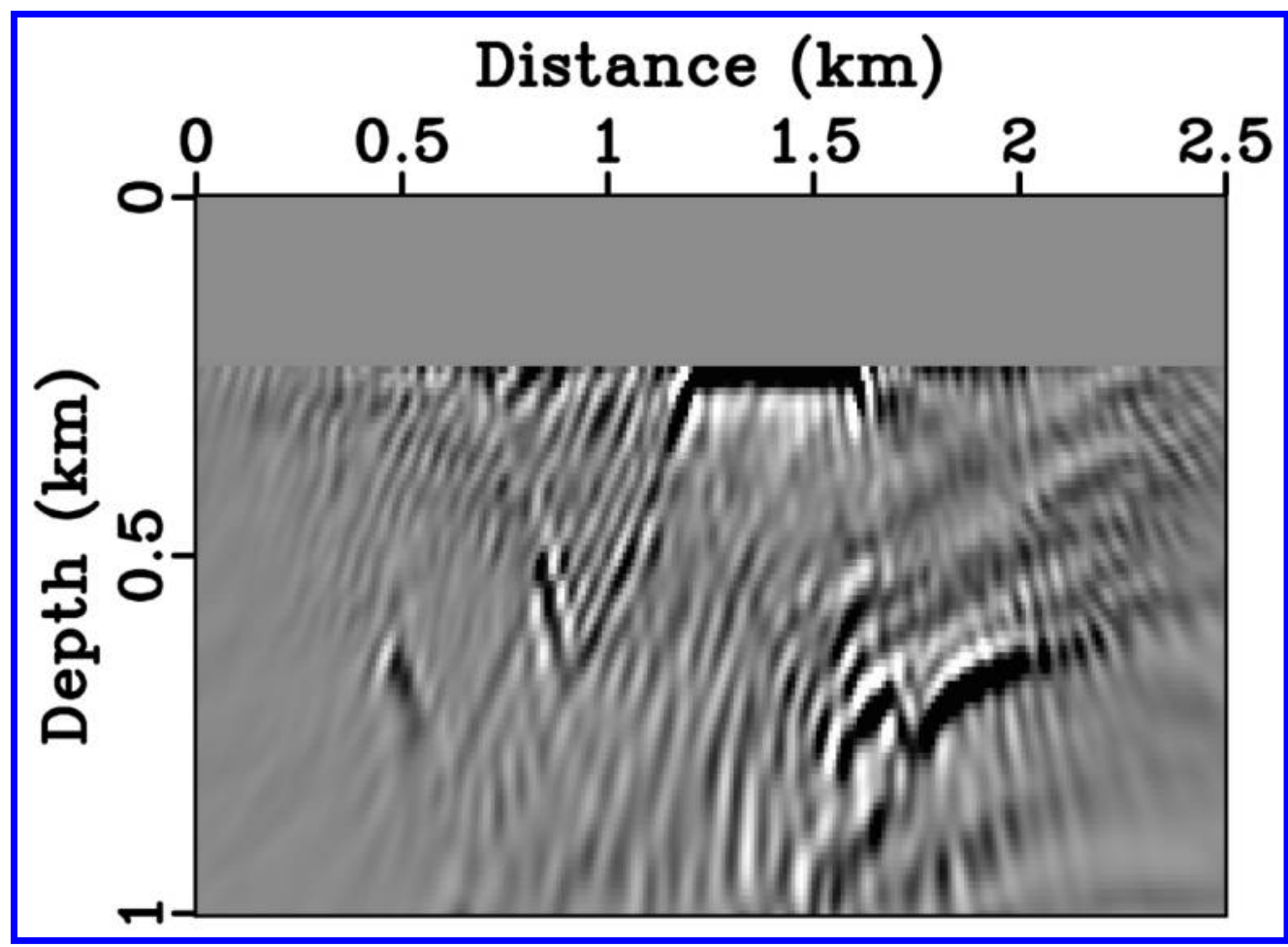

Figure number and letter: Figure 7d. Figure 7 The LS-GIMI images corresponding to (a) $\mathrm{I}_{E}$ and (b) $\mathrm{I}_{E}{ }^{\mathrm{mig}}$ after the first iteration of the least-squares optimization; The final LS-GIMI images (c) $I_{E}$ and (d) $I_{E}{ }^{m i g}$ after 30 iterations. The intense energy in the image $\mathrm{I}_{E}$ is caused by the large impedance contrast at the top of the Marmousi model.

$199 \times 144 \mathrm{~mm}(300 \times 300 \mathrm{DPI})$ 


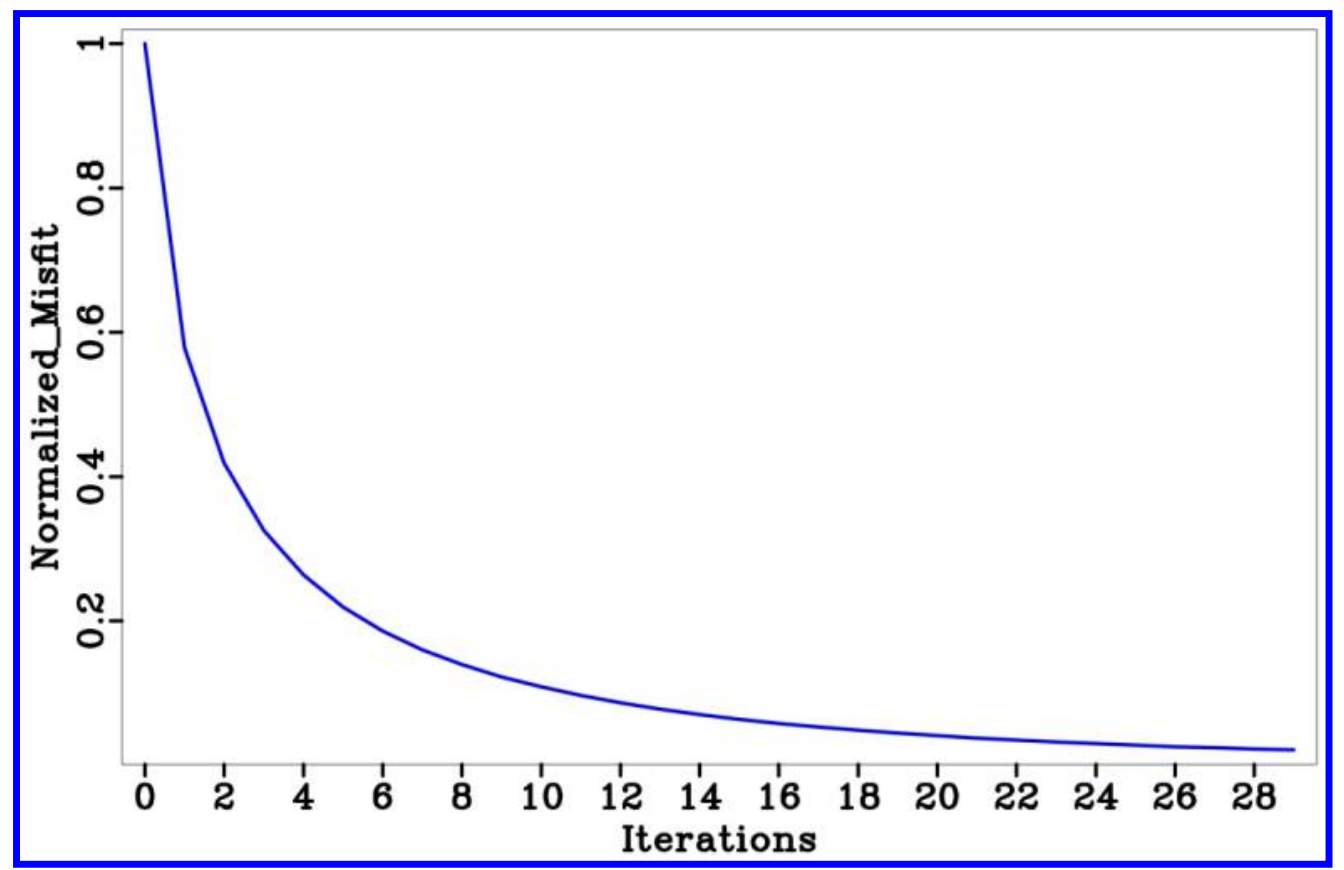

Figure number and letter: Figure 8 . Figure 8 The convergence curve of the objective function for the Marmousi model

$345 \times 223 \mathrm{~mm}(300 \times 300 \mathrm{DPI})$ 


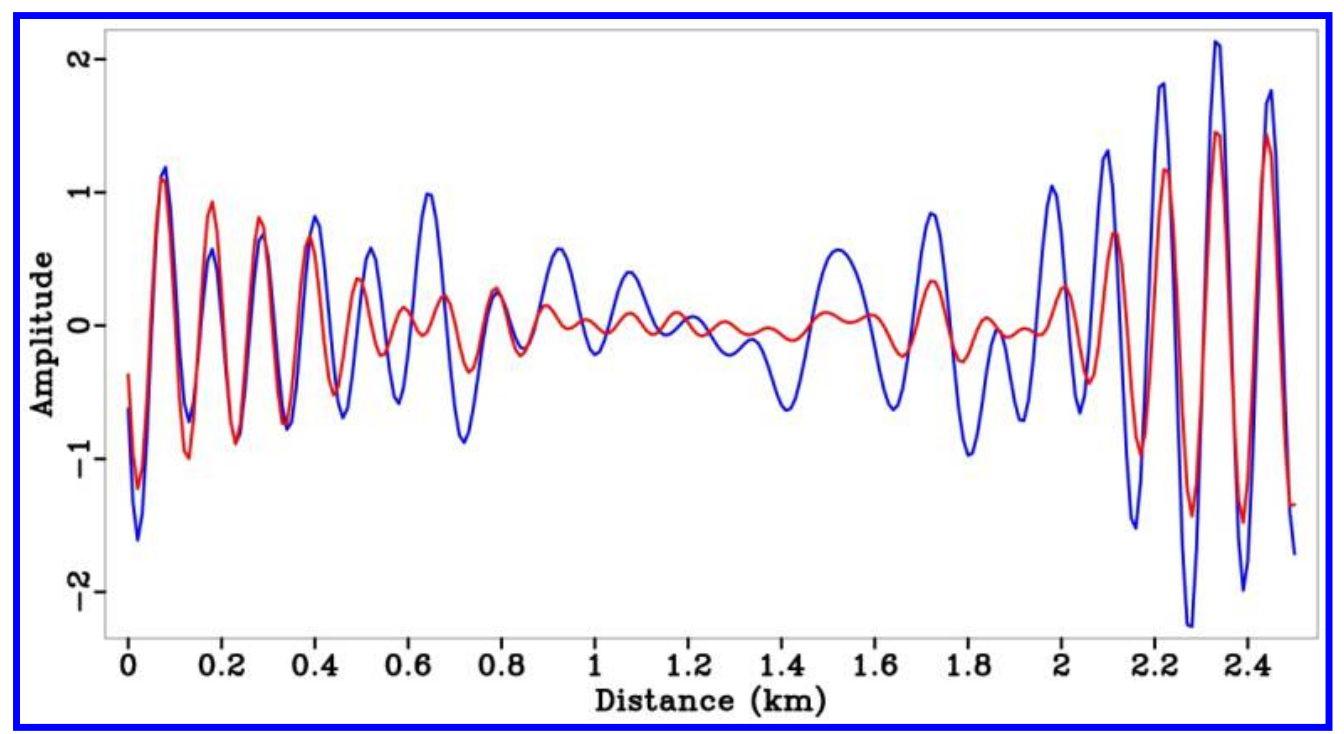

Figure number and letter: Figure 9a. Figure 9 The real part of the original data (red lines) by the true model and the adjoint data (blue lines) by the image in Figure 7c at $15 \mathrm{~Hz}$ (a) and $19 \mathrm{~Hz}$ (b), for 64th shot gather.

$417 \times 225 \mathrm{~mm}(300 \times 300$ DPI $)$ 


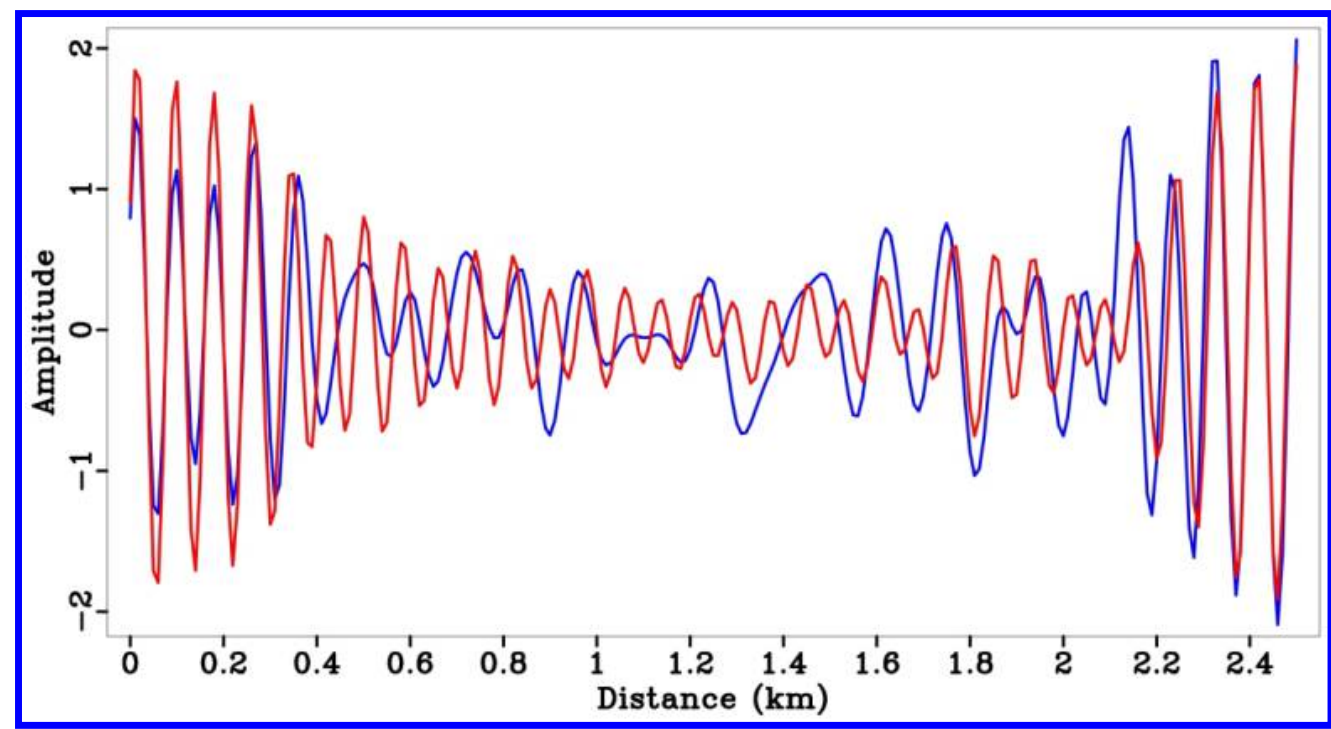

Figure number and letter: Figure $9 \mathrm{~b}$. Figure 9 The real part of the original data (red lines) by the true model and the adjoint data (blue lines) by the image in Figure 7c at $15 \mathrm{~Hz}$ (a) and $19 \mathrm{~Hz}$ (b), for 64th shot gather.

$417 \times 225 \mathrm{~mm}(300 \times 300 \mathrm{DPI})$ 


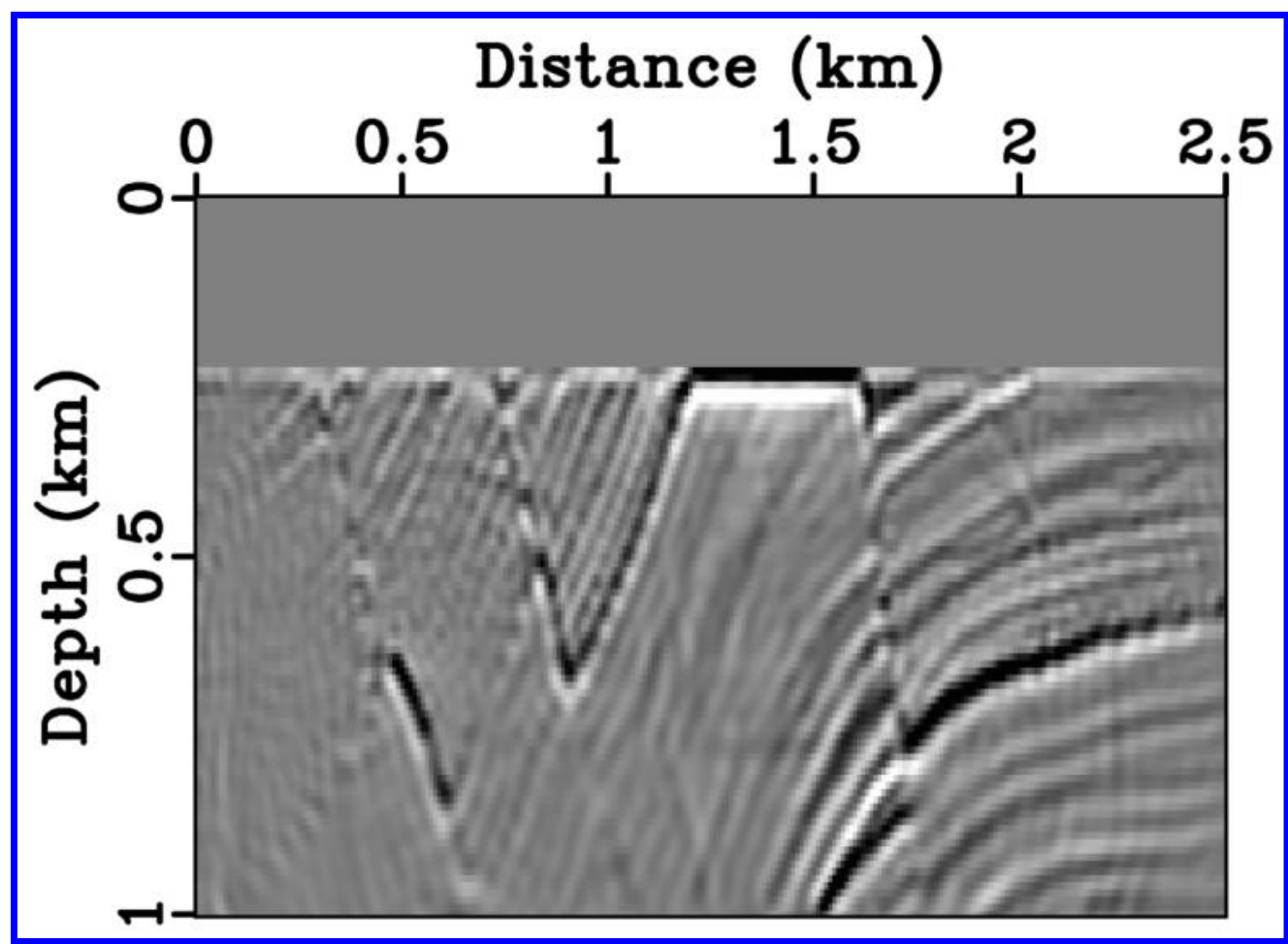

Figure number and letter: Figure 10a. Figure 10 (a) The LS-RTM image of the Marmousi model, (b) the sum of the LS-RTM and LS-GIMI images.

$199 \times 144 \mathrm{~mm}(300 \times 300 \mathrm{DPI})$ 


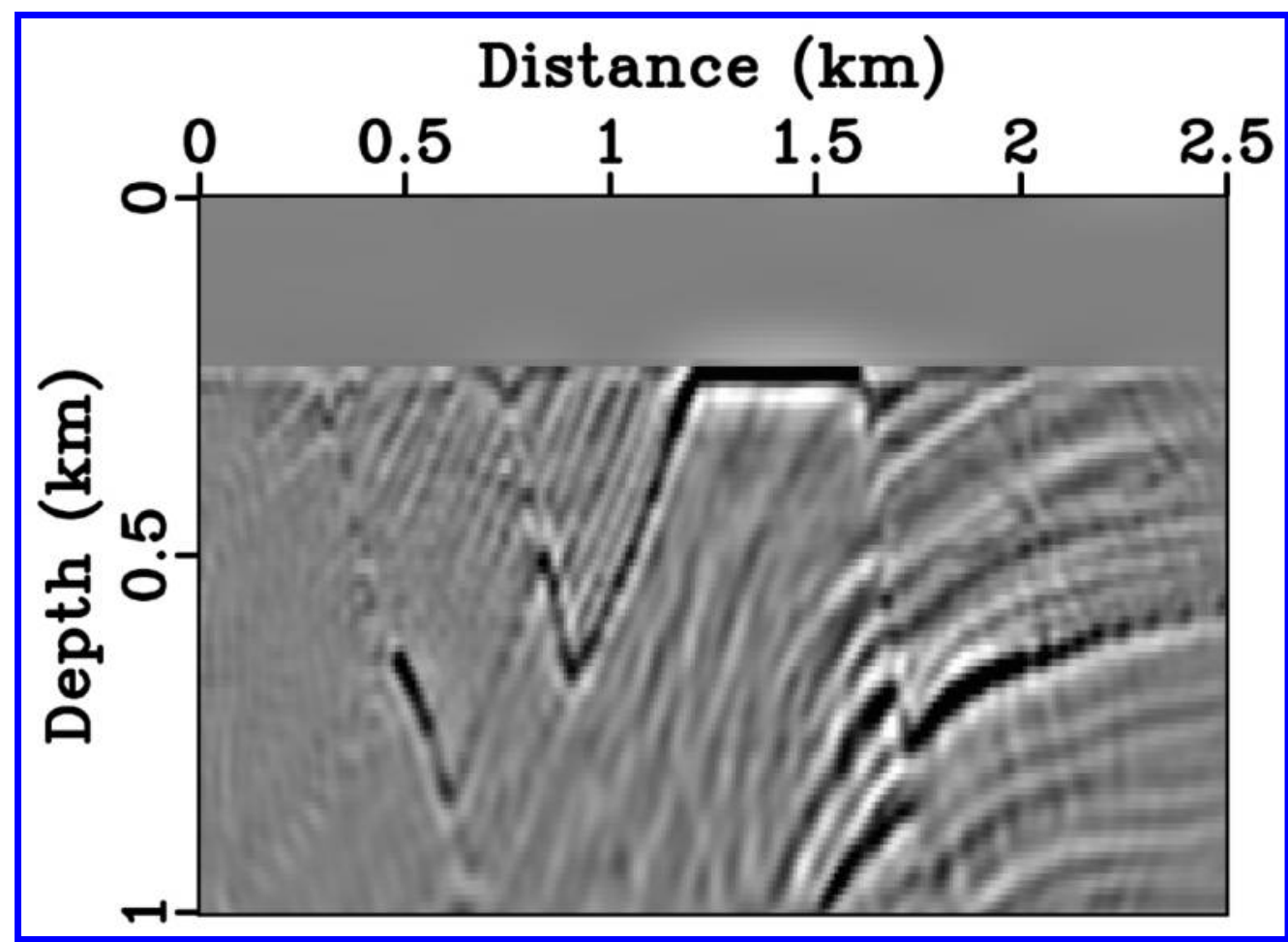

Figure number and letter: Figure 10b. Figure 10 (a) The LS-RTM image of the Marmousi model, (b) the sum of the LS-RTM and LS-GIMI images.

$199 \times 144 \mathrm{~mm}(300 \times 300$ DPI) 
DATA AND MATERIALS AVAILABILITY

Data associated with this research are available and can be obtained by contacting the corresponding author. 